\title{
Molecular characterization of gaseous and particulate oxygenated compounds at a remote site in Cape Corsica in the western Mediterranean basin.
}

Vincent Michoud ${ }^{1}$, Elise Hallemans ${ }^{1,2}$, Laura Chiappini ${ }^{2}$, Eva Leoz-Garziandia ${ }^{2}$, Aurélie Colomb ${ }^{3}$, Sébastien Dusanter ${ }^{4}$, Isabelle Fronval ${ }^{4}$, François Gheusi $^{5}$, Jean-Luc Jaffrezo ${ }^{6}$, Thierry Léonardis ${ }^{4}$, Nadine Locoge ${ }^{4}$, Nicolas Marchand ${ }^{7}$, Stéphane Sauvage ${ }^{4}$, Jean Sciare ${ }^{8,9}$, Jean-François Doussin $^{1}$

[1] LISA, UMR CNRS 7583, Université de Paris, Université Paris-Est-Créteil, Institut Pierre Simon Laplace (IPSL), Créteil, France

[2] Institut National de l'Environnement Industriel et des Risques, Verneuil-en-Halatte, France

[3] LaMP, CNRS UMR6016, Clermont Université, Université Blaise Pascal, Aubière, France

[4] IMT Lille Douai, Univ. Lille, SAGE - Département Sciences de l'Atmosphère et Génie de l'Environnement, 59000 Lille, France

[5] Laboratoire d'Aérologie, Université de Toulouse, CNRS, Toulouse, France

[6] Université Grenoble Alpes, CNRS, IRD, IGE, 38000 Grenoble, France

[7] Aix Marseille Univ, CNRS, LCE, Marseille, 13003, France

[8] LSCE, CNRS-CEA-UVSQ, IPSL, Université Paris-Saclay, Gif-sur-Yvette, France

[9] EEWRC, The Cyprus Institute, Nicosia, Cyprus 
DMM : Derivatized Molar Mass ; MM : Molar Mass ; MS : Mass Spectra ; RT : Retention Time ; QL : Quantification Limit ; ND : Not Detected

\begin{tabular}{|c|c|c|c|c|c|c|c|c|c|c|c|c|c|}
\hline \multicolumn{8}{|c|}{ Identification } & \multicolumn{6}{|c|}{ Quantification } \\
\hline \multirow{2}{*}{ Main Fragments } & \multirow{2}{*}{ DMM } & \multirow{2}{*}{$\begin{array}{c}\text { Number } \\
\text { of } \\
\text { functions }\end{array}$} & \multirow[b]{2}{*}{ MM } & \multirow{2}{*}{ Name } & \multirow[b]{2}{*}{ Structure } & \multirow{2}{*}{$\begin{array}{c}\mathrm{O} / \mathrm{C} \\
\text { Log (Saturation Vapor } \\
\text { Pressure) }\end{array}$} & \multirow{2}{*}{ Identification methods } & \multirow{2}{*}{ Substitut } & \multirow{2}{*}{ External standard } & \multicolumn{2}{|c|}{ Gaseous Phase } & \multicolumn{2}{|c|}{ Particulate phase } \\
\hline & & & & & & & & & & $\begin{array}{c}\mathrm{m} / \mathrm{z} \\
\text { integrated }\end{array}$ & $\begin{array}{c}\text { Concentration } \\
\left(\mathrm{ng} / \mathrm{m}^{3}\right)\end{array}$ & $\begin{array}{c}\mathrm{m} / \mathrm{z} \\
\text { integrated }\end{array}$ & $\begin{array}{c}\text { Concentration } \\
\left(\mathrm{ng} / \mathrm{m}^{3}\right)\end{array}$ \\
\hline \multicolumn{14}{|c|}{ Carbonyl Compounds } \\
\hline EI : $117,161,181,236$ & 253 & 1 & 58 & Propanal & & $\begin{array}{l}0.33 \\
10^{-0.5}\end{array}$ & $\begin{array}{c}\text { Standard } \\
\text { Rossignol (2012) } \\
\text { Müller et al. (2006) }\end{array}$ & Butanal-d8 & Pentanal & 181 & $200 \pm 69$ & 181 & $10 \pm 1$ \\
\hline $\begin{array}{l}\text { EI : } 117,181,195,197, \\
198,251\end{array}$ & 251 & 1 & 56 & Acroleine & & $\begin{array}{l}0.33 \\
10^{-0.7}\end{array}$ & $\begin{array}{c}\text { Standard } \\
\text { Rossignol (2012) } \\
\text { Jaoui et al. (2014) }\end{array}$ & 3-Methylbutanal-d2 & Methacrolein & 181 & $734 \pm 125$ & DMM & $6 \pm 1$ \\
\hline EI : $43,181,239,250,267$ & 267 & 1 & 72 & Methylpropanal & & $\begin{array}{l}0.25 \\
10^{-0.8}\end{array}$ & $\begin{array}{c}\mathrm{MS} / \mathrm{RT} \\
\text { Rossignol (2012) }\end{array}$ & 3-Methylbutanal-d2 & 2-Ethylbutanal & ND & $\mathrm{ND}$ & 43 & $1 \pm 0,4$ \\
\hline EI : 43, $57,181,195,250$ & 267 & 1 & 72 & $\begin{array}{l}\text { 2-Butanone (= Methyl } \\
\text { Ethyl Ketone) }\end{array}$ & & $\begin{array}{l}0.25 \\
10^{-0.8}\end{array}$ & $\begin{array}{l}\text { Standard } \\
\text { Müller et al. (2006) }\end{array}$ & 2-Hexanone-d5 & 2-Hexanone-d5 & DMM - 181 & $230 \pm 18$ & DMM & blank \\
\hline EI : $41,69,181,265$ & 265 & 1 & 75 & Methacrolein & & $\begin{array}{l}0.25 \\
10^{-1.0}\end{array}$ & $\begin{array}{l}\text { Standard } \\
\text { Healy et al. (2008) }\end{array}$ & 3-Methylbutanal-d2 & Methacrolein & DMM & $230 \pm 16$ & DMM & $2 \pm 1$ \\
\hline $\begin{array}{l}\text { EI : 42, 56, 69, 99, 117, } \\
181,195,264,265\end{array}$ & 265 & 1 & 75 & $\begin{array}{l}\text { Methyl Vinyl Ketone } \\
\text { (= 3-Buten-2-one) }\end{array}$ & & $\begin{array}{l}0.25 \\
10^{-1.0}\end{array}$ & $\begin{array}{c}\text { Standard } \\
\text { Healy et al. (2008) }\end{array}$ & 2-Hexanone-d5 & 3-Buten-2-one & 181 & $539 \pm 30$ & DMM & $3 \pm 0,5$ \\
\hline $\begin{array}{l}\text { EI : } 41,56,131,181,195, \\
235,264\end{array}$ & 267 & 1 & 72 & Butanal & & $\begin{array}{l}0.25 \\
10^{-0.9}\end{array}$ & $\begin{array}{l}\text { Standard } \\
\text { Müller et al. (2006) }\end{array}$ & Butanal-d8 & Pentanal & 181 & $88 \pm 75$ & 181 & blank \\
\hline $\begin{array}{l}\text { EI: } 58,100,181,221,253, \\
281 \\
\text { CI }: 282,310,322\end{array}$ & 281 & 1 & 86 & 3-Methyl-2-butanone & & $\begin{array}{c}0.2 \\
10^{-1.2}\end{array}$ & $\begin{array}{c}\text { MS/RT/Nist } \\
\text { Rossignol (2012) }\end{array}$ & 2-Hexanone-d5 & 2-Hexanone-d5 & ND & ND & DMM & $1 \pm 1$ \\
\hline $\begin{array}{l}\text { EI: } 46,93,117,181,195, \\
218,281\end{array}$ & 281 & 1 & 86 & 3-Pentanone & & $\begin{array}{c}0.2 \\
10^{-1.3}\end{array}$ & $\begin{array}{c}\text { MS/RT } \\
\text { Rossignol (2012) }\end{array}$ & 2-Hexanone-d5 & 2-Butanone & 181 & $<\mathrm{QL}$ & ND & ND \\
\hline $\begin{array}{l}\text { II: } 43,71,167,181,195, \\
252,264,281, \\
\text { CI: } 282,310,322\end{array}$ & 281 & 1 & 86 & 2-Methylbutanal & & $\begin{array}{c}0.2 \\
10^{-1.3}\end{array}$ & $\mathrm{MS} / \mathrm{RT}$ & Butanal-d8 & Pentanal & ND & ND & 181 & $1 \pm 1$ \\
\hline $\begin{array}{l}\text { EI : } 41,55,69,161,181, \\
222,239\end{array}$ & 281 & 1 & 86 & Pentanal & & $\begin{array}{c}0.2 \\
10^{-1.4}\end{array}$ & $\begin{array}{l}\text { Standard } \\
\text { Müller et al. (2006) }\end{array}$ & Butanal-d8 & Pentanal & 181 & $303 \pm 129$ & DMM - 42 & $2 \pm 0,4$ \\
\hline $\begin{array}{l}\text { EI : } 72,94,117,181,197, \\
236,253,277\end{array}$ & 295 & 1 & 100 & 2-Hexanone & & $\begin{array}{l}0.17 \\
10^{-1.7}\end{array}$ & $\begin{array}{c}\text { Standard } \\
\text { Berndt et al. (2003) }\end{array}$ & 2-Hexanone-d5 & 2-Hexanone & DMM - 42 & $85 \pm 4$ & 72 & blank \\
\hline $\begin{array}{l}\text { EI : } 43,181,278,293 \\
\text { CI : } 294,322,334\end{array}$ & 293 & 1 & 98 & 4-Hexen-3-one & & $\begin{array}{l}0.17 \\
10^{-2.0}\end{array}$ & $\mathrm{MS} / \mathrm{RT} / \mathrm{Nist}$ & 1 & 1 & ND & ND & 181 & blank \\
\hline
\end{tabular}


Identification and quantification of compounds during ChArMEx campaign.

DMM : Derivatized Molar Mass ; MM : Molar Mass ; MS : Mass Spectra ; RT : Retention Time ; QL : Quantification Limit ; ND : Not Detected

\begin{tabular}{|c|c|c|c|c|c|c|c|c|c|c|c|c|c|}
\hline \multicolumn{8}{|c|}{ Identification } & \multicolumn{6}{|c|}{ Quantification } \\
\hline \multirow[b]{2}{*}{ Main Fragments } & \multirow[b]{2}{*}{ DMM } & \multirow{2}{*}{$\begin{array}{l}\text { Number } \\
\text { of } \\
\text { functions }\end{array}$} & \multirow[b]{2}{*}{ MM } & \multirow[b]{2}{*}{ Name } & \multirow[b]{2}{*}{ Structure } & \multirow{2}{*}{$\begin{array}{c}\mathrm{O} / \mathrm{C} \\
\begin{array}{l}\log (\text { Saturation Vapor } \\
\text { Pressure) }\end{array}\end{array}$} & \multirow[b]{2}{*}{ Identification methods } & \multirow[b]{2}{*}{ Substitut } & \multirow[b]{2}{*}{ External standard } & \multicolumn{2}{|c|}{ Gaseous Phase } & \multicolumn{2}{|c|}{ Particulate phase } \\
\hline & & & & & & & & & & $\begin{array}{c}\mathrm{m} / \mathrm{z} \\
\text { integrated }\end{array}$ & $\begin{array}{c}\text { Concentration } \\
\left(\mathrm{ng} / \mathrm{m}^{3}\right)\end{array}$ & $\underset{\text { integrated }}{\mathrm{m} / \mathrm{z}}$ & $\begin{array}{c}\text { Concentration } \\
\left(\mathrm{ng} / \mathrm{m}^{3}\right)\end{array}$ \\
\hline $\begin{array}{l}\text { EI : } 43,181,278,293 \\
\text { CI : } 294,322,334\end{array}$ & 293 & 1 & 98 & 5-Hexen-3-one & & $\begin{array}{l}0.17 \\
10^{-1.7}\end{array}$ & MS/RT/Nist & 1 & 1 & ND & ND & 181 & blank \\
\hline $\begin{array}{l}\text { EI : 43, 98, 117, 131, 161, } \\
181,264,279\end{array}$ & 279 & 1 & 84 & 3-Methyl-2-butenal & & $\begin{array}{l}0.2 \\
10^{-1.5}\end{array}$ & Standard & 3-Methylbutanal-d2 & 3-Methyl-2-butenal & DMM - 15 & $<\mathrm{QL}$ & 181 & $<\mathrm{QL}$ \\
\hline $\begin{array}{l}\text { EI : } 41,55,72,86,181, \\
230,267,309\end{array}$ & 309 & 1 & 114 & 3-Heptanone & & $\begin{array}{l}0.14 \\
10^{-2.2}\end{array}$ & $\begin{array}{c}\text { MS/RT/Nist } \\
\text { Rossignol (2012) }\end{array}$ & 1 & I & $\mathrm{ND}$ & $\mathrm{ND}$ & DMM & $<\mathrm{QL}$ \\
\hline $\begin{array}{l}\text { EI }: 41,66,82,114,161 \\
181,239\end{array}$ & 295 & 1 & 100 & Hexanal & & $\begin{array}{l}0.17 \\
10^{-1.8}\end{array}$ & $\begin{array}{c}\text { MS/RT/Nist } \\
\text { Rossignol (2012) }\end{array}$ & Butanal-d8 & Pentanal & 181 & $311 \pm 247$ & 181 & $3 \pm 0,5$ \\
\hline $\begin{array}{l}\text { EI } 41,99,161,181,222, \\
239,252,295\end{array}$ & 295 & 1 & 100 & 4-Methylpentanal & & $\begin{array}{l}0.17 \\
10^{-1.7}\end{array}$ & MS/RT/Nist & 2-Hexanone-d5 & 2-Ethylbutanal & $\mathrm{ND}$ & $\mathrm{ND}$ & 43 & $20 \pm 1$ \\
\hline $\begin{array}{l}\text { EI : } 128,181,253,309 \\
\text { C: }: 310,338,350\end{array}$ & 309 & 1 & 114 & 2-Heptanone & & $\begin{array}{l}0.14 \\
10^{-2.2}\end{array}$ & $\begin{array}{c}\text { MS/RT/Nist } \\
\text { Rossignol (2012) }\end{array}$ & 2-Hexanone-d5 & 4-Heptanone & ND & $\mathrm{ND}$ & DMM & $<\mathrm{QL}$ \\
\hline EI : $62,110,181,248,291$ & 291 & 1 & 96 & 2-Furaldehyde & & $\begin{array}{c}0.4 \\
10^{-2.1}\end{array}$ & MS/RT/Nist & Benzaldehyde-d6 & Pentanal & DMM & blank & ND & ND \\
\hline $\begin{array}{l}\text { EI }: 41,67,82,112,161 \\
181,263,276,293\end{array}$ & 293 & 1 & 98 & Cyclohexanone & & $\begin{array}{l}0.17 \\
10^{-2.1}\end{array}$ & $\begin{array}{c}\text { MS/RT/Nist } \\
\text { Rossignol (2012) } \\
\text { Hamilton et al. (2004) }\end{array}$ & Acetophenone-d8 & 2-Butanone & 181 & $744 \pm 109$ & 181 & blank \\
\hline $\begin{array}{l}\text { EI : 41, } 55,69,99,128, \\
161,181,222,239\end{array}$ & 309 & 1 & 114 & Heptanal & & $\begin{array}{l}0.14 \\
10^{-2.3}\end{array}$ & $\begin{array}{c}\text { Standard } \\
\text { Müller et al. (2006) }\end{array}$ & Butanal-d8 & Pentanal & 181 & $421 \pm 122$ & 181 & $2 \pm 0,5$ \\
\hline $\begin{array}{l}\text { EI : } 51,65,77,103,106 \\
167,181,258,315\end{array}$ & 315 & 1 & 120 & m-Tolualdehyde & & $\begin{array}{l}0.13 \\
10^{-3.3}\end{array}$ & MS/RT/Nist & Acetophenone-d8 & 3-Methyl-2-butenal & DMM & $407 \pm 47$ & ND & ND \\
\hline $\begin{array}{l}\text { EI : } 41,55,69,82,99,124 \\
142,169,181,222,239\end{array}$ & 323 & 1 & 128 & Octanal & & $\begin{array}{l}0.13 \\
10^{-2.7}\end{array}$ & $\begin{array}{c}\text { MS/RT/Nist } \\
\text { Rossignol (2012) } \\
\text { Müller et al. (2006) }\end{array}$ & Butanal-d8 & Pentanal & ND & ND & 181 & blank \\
\hline $\begin{array}{l}\text { EI : } 41,55,72,117,236, \\
253,280,322 \\
\text { CI : }: 338,366,378\end{array}$ & 337 & 1 & 142 & $\begin{array}{l}\text { 2,6-Dimethyl-4- } \\
\text { heptanone }\end{array}$ & & $\begin{array}{l}0.11 \\
10^{-2.9}\end{array}$ & $\mathrm{MS} / \mathrm{RT}$ & 2-Hexanone-d5 & 4-Heptanone & $\mathrm{ND}$ & $\mathrm{ND}$ & 181 & $1 \pm 1$ \\
\hline $\begin{array}{l}\text { EI : } 39,51,65,77,89,181 \text {, } \\
271,301\end{array}$ & 301 & 1 & 106 & Benzaldehyde & & $\begin{array}{l}0.14 \\
10^{-2.8}\end{array}$ & $\begin{array}{c}\text { Standard } \\
\text { Müller et al. (2006) }\end{array}$ & Benzaldehyde-d6 & Pentanal & 181 & $586 \pm 144$ & 181 & blank \\
\hline $\begin{array}{l}\text { EI : } 41,50,77,106,181 \\
258,315\end{array}$ & 315 & 1 & 120 & Acetophenone & & $\begin{array}{l}0.13 \\
10^{-3.1}\end{array}$ & $\begin{array}{c}\text { Standard } \\
\text { Hamilton et al. (2004) }\end{array}$ & Acetophenone-d8 & 2-Butanone & $\mathrm{ND}$ & $\mathrm{ND}$ & 181 & blank \\
\hline
\end{tabular}


Identification and quantification of compounds during ChArMEx campaign.

DMM : Derivatized Molar Mass ; MM : Molar Mass ; MS : Mass Spectra ; RT : Retention Time ; QL : Quantification Limit ; ND : Not Detected

\begin{tabular}{|c|c|c|c|c|c|c|c|c|c|c|c|c|c|}
\hline \multicolumn{8}{|c|}{ Identification } & \multicolumn{6}{|c|}{ Quantification } \\
\hline \multirow[b]{2}{*}{ Main Fragments } & \multirow[b]{2}{*}{ DMM } & \multirow{2}{*}{$\begin{array}{l}\text { Number } \\
\text { of } \\
\text { functions }\end{array}$} & \multirow[b]{2}{*}{ MM } & \multirow[b]{2}{*}{ Name } & \multirow[b]{2}{*}{ Structure } & \multirow{2}{*}{$\begin{array}{c}\mathrm{O} / \mathrm{C} \\
\begin{array}{c}\log (\text { Saturation Vapor } \\
\text { Pressure) }\end{array}\end{array}$} & \multirow{2}{*}{ Identification method } & \multirow[b]{2}{*}{ Substitut } & \multirow[b]{2}{*}{ External standard } & \multicolumn{2}{|c|}{ Gaseous Phase } & \multicolumn{2}{|c|}{ Particulate phase } \\
\hline & & & & & & & & & & $\begin{array}{c}\mathrm{m} / \mathrm{z} \\
\text { integrated }\end{array}$ & $\begin{array}{c}\text { Concentration } \\
\left(\mathrm{ng} / \mathrm{m}^{3}\right)\end{array}$ & $\begin{array}{c}\mathrm{m} / \mathrm{z} \\
\text { integrated }\end{array}$ & $\begin{array}{c}\text { Concentration } \\
\left(\mathrm{ng} / \mathrm{m}^{3}\right)\end{array}$ \\
\hline $\begin{array}{l}\text { EI : } 41,55,69,81,99,117 \\
156,161,181,222,239\end{array}$ & 337 & 1 & 142 & Nonanal & & $\begin{array}{l}0.11 \\
10^{-3.2}\end{array}$ & $\begin{array}{c}\text { MS/RT/Nist } \\
\text { Rossignol (2012) } \\
\text { Matsunaga et al. (2003) }\end{array}$ & Butanal-d8 & Pentanal & $\mathrm{ND}$ & $\mathrm{ND}$ & 181 & blank \\
\hline $\begin{array}{l}\text { EI: } 181,221,253,276, \\
290\end{array}$ & 333 & 1 & 138 & Nopinone & & $\begin{array}{l}0.11 \\
10^{-3.3}\end{array}$ & $\begin{array}{c}\text { Standard } \\
\text { Cahill et al, (2006) }\end{array}$ & 2-Hexanone-d5 & Nopinone & DMM - 43 & $673 \pm 44$ & DMM - 43 & $3 \pm 0,3$ \\
\hline $\begin{array}{l}\text { EI : } 41,79,95,110,152, \\
181,290, \\
\text { CI }: 334,362,374\end{array}$ & 333 & 1 & 138 & Ketolimonene & & $\begin{array}{l}0.11 \\
10^{-3.2}\end{array}$ & $\begin{array}{c}\text { MS/RT } \\
\text { Rossignol (2012) }\end{array}$ & Acetophenone-d8 & 2-Butanone & 181 & $<\mathrm{QL}$ & ND & ND \\
\hline $\begin{array}{l}\text { EI : } 41,55,69,83,99,117, \\
161,181,222,239\end{array}$ & 351 & 1 & 156 & Decanal & & $\begin{array}{l}0.10 \\
10^{-3.6}\end{array}$ & $\begin{array}{c}\text { MS/RT/Nist } \\
\text { Rossignol (2012) } \\
\text { Matsunaga et al. (2003) }\end{array}$ & Butanal-d8 & Pentanal & 181 & $2809 \pm 192$ & 181 & $1 \pm 0,5$ \\
\hline $\begin{array}{l}\text { EI: } 99,117,161,181,195, \\
239,448\end{array}$ & 448 & 2 & 58 & Glyoxal & & $\begin{array}{c}1.0 \\
10^{-1.6}\end{array}$ & $\begin{array}{l}\text { Standard } \\
\text { Matsunaga et al. (2004) }\end{array}$ & 2,3-Butanedione-d6 & Glyoxal & DMM & $3017 \pm 184$ & DMM - 197 & $1 \pm 0,2$ \\
\hline $\begin{array}{l}\text { EI : } 41,55,161,181,195, \\
222,239,265,462\end{array}$ & 462 & 2 & 72 & Methylglyoxal & & $\begin{array}{l}0.67 \\
10^{-1.2}\end{array}$ & $\begin{array}{c}\text { Standard } \\
\text { Matsunaga et al. (2004) }\end{array}$ & 2,3-Butanedione-d6 & Methylglyoxal & DMM - 197 & $700 \pm 69$ & DMM - 197 & $13 \pm 1$ \\
\hline $\begin{array}{l}\text { EI : } 42,99,161,181,195 \text {, } \\
279,446,476\end{array}$ & 476 & 2 & 86 & Dimethylglyoxal & & $\begin{array}{c}0.5 \\
10^{-1.6}\end{array}$ & $\begin{array}{c}\text { Standard } \\
\text { Rossignol (2012) } \\
\text { Jaoui et al, (2012) }\end{array}$ & 2,3-Butanedione-d6 & Dimethylglyoxal & DMM - 197 & $286 \pm 63$ & 181 & $9 \pm 1$ \\
\hline $\begin{array}{l}\text { EI : } 42,82,161,181,195, \\
208,252,279,293,309, \\
490\end{array}$ & 490 & 2 & 100 & 4-Oxopentanal & & $\begin{array}{c}0.4 \\
10^{-2.2} \\
\end{array}$ & $\begin{array}{l}\text { Standard } \\
\text { Matsunaga et al. (2004) }\end{array}$ & 2,3-Butanedione-d6 & 4-Oxopentanal & DMM - 181 & $3903 \pm 182$ & $\mathrm{ND}$ & $\mathrm{ND}$ \\
\hline \multicolumn{14}{|c|}{ Hydroxyl compounds and carboxylic acids } \\
\hline $\begin{array}{l}\text { EI : } 45,55,73,75,85,99, \\
129,171\end{array}$ & 186 & 1 & 72 & 2-Propenoic acid & & $\begin{array}{l}0.67 \\
10^{-2.8}\end{array}$ & MS/RT/Nist & Pentanoic-d9 acid & Methacrylic acid & DMM - 57 & $211 \pm 106$ & $\mathrm{ND}$ & $\mathrm{ND}$ \\
\hline EI : $75,115,131,149,173$ & 188 & 1 & 74 & Glyoxilic acid & & $\begin{array}{c}1.5 \\
10^{-5.6}\end{array}$ & $\begin{array}{c}\text { Standard } \\
\text { Cahill et al, (2006) }\end{array}$ & Pentanoic-d9 acid & Methacrylic acid & DMM - 57 & $1042 \pm 225$ & $\mathrm{ND}$ & $\mathrm{ND}$ \\
\hline $\begin{array}{l}\text { EI : } 45,75,115,131,149, \\
173\end{array}$ & 188 & 1 & 74 & Propanoic acid & & $\begin{array}{l}0.67 \\
10^{-2.5}\end{array}$ & $\begin{array}{c}\text { MS/RT/Nist } \\
\text { Cahill et al, (2006) }\end{array}$ & Heptanoic acid -d13 & Heptanoic acid & DMM - 57 & $862 \pm 106$ & $\mathrm{ND}$ & ND \\
\hline EI : $41,69,99,143,185$ & 200 & 1 & 86 & Methacrylic acid & & $\begin{array}{c}0.5 \\
10^{-3.2}\end{array}$ & Standard & Pentanoic-d9 acid & Methacrylic acid & DMM - 57 & $128 \pm 111$ & DMM - 57 & $0,4 \pm 1$ \\
\hline
\end{tabular}


Identification and quantification of compounds during ChArMEx campaign.

DMM : Derivatized Molar Mass ; MM : Molar Mass ; MS : Mass Spectra ; RT : Retention Time ; QL : Quantification Limit ; ND : Not Detected

\begin{tabular}{|c|c|c|c|c|c|c|c|c|c|c|c|c|c|}
\hline \multicolumn{8}{|c|}{ Identification } & \multicolumn{6}{|c|}{ Quantification } \\
\hline \multirow{2}{*}{ Main Fragments } & \multirow[b]{2}{*}{ DMM } & \multirow{2}{*}{$\begin{array}{l}\text { Number } \\
\text { of } \\
\text { functions }\end{array}$} & \multirow[b]{2}{*}{ мM } & \multirow{2}{*}{ Name } & \multirow[b]{2}{*}{ Structure } & \multirow{2}{*}{$\begin{array}{c}\mathrm{O} / \mathrm{C} \\
\begin{array}{c}\log (\text { Saturation Vapor } \\
\text { Pressure) }\end{array}\end{array}$} & \multirow[b]{2}{*}{ Identification method } & \multirow[b]{2}{*}{ Substitut } & \multirow{2}{*}{ External standard } & \multicolumn{2}{|c|}{ Gaseous Phase } & \multicolumn{2}{|c|}{ Particulate phase } \\
\hline & & & & & & & & & & $\begin{array}{c}\mathrm{m} / \mathrm{z} \\
\text { integrated }\end{array}$ & $\begin{array}{c}\text { Concentration } \\
\left(\mathrm{ng} / \mathrm{m}^{3}\right)\end{array}$ & $\begin{array}{c}\mathrm{m} / \mathrm{z} \\
\text { integrated }\end{array}$ & $\begin{array}{c}\text { Concentration } \\
\left(\mathrm{ng} / \mathrm{m}^{3}\right)\end{array}$ \\
\hline $\begin{array}{l}\text { EI : } 43,73,75,103,115 \text {, } \\
145\end{array}$ & 202 & 1 & 88 & Pyruvic acid & & $\begin{array}{c}1 \\
10^{-3.6}\end{array}$ & $\begin{array}{c}\text { Standard } \\
\text { Kawamura et Yasui } \\
\text { (2005) }\end{array}$ & Pentanoic-d9 acid & Pyruvic acid & DMM - 57 & interferences & DMM - 57 & $0,3 \pm 1$ \\
\hline EI $: 41,75,99,143,185$ & 200 & 1 & 86 & Crotonic acid & & $\begin{array}{c}0.5 \\
10^{-3.3}\end{array}$ & Standard & Pentanoic-d9 acid & Crotonic acid & DMM - 57 & $<\mathrm{QL}$ & DMM - 57 & $2 \pm 0,5$ \\
\hline $\begin{array}{l}\text { EI : } 41,58,75,115,129 \\
159,201\end{array}$ & 216 & 1 & 102 & Pentanoic acid & & $\begin{array}{c}0.4 \\
10^{-3.5}\end{array}$ & Étalon & Heptanoic acid -d13 & Heptanoic acid & DMM - 57 & $483 \pm 70$ & DMM - 57 & blank \\
\hline $\begin{array}{l}\text { EE : } 47,58,75,115,129, \\
173\end{array}$ & 230 & 1 & 116 & 3-Ethylbutyrique acid & & $\begin{array}{l}0.33 \\
10^{-3.9}\end{array}$ & $\mathrm{MS} / \mathrm{RT}$ & Pentanoic-d9 acid & 2-Ethylbutyric acid & DMM - 57 & $118 \pm 55$ & ND & ND \\
\hline EI : $41,75,131,173,215$ & 230 & 1 & 116 & Hexanoic acid & & $\begin{array}{l}0.33 \\
10^{-4.0}\end{array}$ & $\begin{array}{c}\text { MS/RT/Nist } \\
\text { Rossignol (2012) }\end{array}$ & Heptanoic acid -d13 & Heptanoic acid & DMM - 57 & $722 \pm 73$ & DMM - 57 & blank \\
\hline $\begin{array}{l}\text { EI : } 43,75,99,129,145, \\
155,173,181,215\end{array}$ & 230 & 1 & 116 & Levulinic acid & & $\begin{array}{c}0.6 \\
10^{-4.6}\end{array}$ & $\begin{array}{l}\text { standard } \\
\text { Jaoui et al. (2006) }\end{array}$ & Heptanoic acid -d13 & Levulinic acid & DMM -85 & $606 \pm 91$ & DMM - 57 & $10 \pm 1$ \\
\hline $\begin{array}{l}\text { EI }: 41,75,95,116,131 \\
187,229\end{array}$ & 244 & 1 & 130 & Heptanoic acid & & $\begin{array}{l}0.29 \\
10^{-4.5}\end{array}$ & $\begin{array}{c}\text { Standard } \\
\text { Rossignol (2012) }\end{array}$ & Heptanoic acid -d13 & Heptanoic acid & 131 & $399 \pm 48$ & DMM - 57 & blank \\
\hline $\begin{array}{l}\text { EI : } 41,57,73,85,103, \\
133,147,189,219,247\end{array}$ & 304 & 2 & 76 & Glycolic acid & & $\begin{array}{c}1.5 \\
10^{-4.6}\end{array}$ & $\begin{array}{c}\text { Standard } \\
\text { Cahill et al, (2006) }\end{array}$ & Heptanoic acid -d13 & Glycolic acid & 147 & $201 \pm 67$ & 115 & $19 \pm 2$ \\
\hline $\begin{array}{l}\text { EI: } 41,57,73,115,133, \\
1477,189,217,247,275, \\
317\end{array}$ & 332 & 2 & 104 & $\begin{array}{l}\text { 3,3-dihydroxy-2- } \\
\text { butanone }\end{array}$ & & $\begin{array}{l}0.75 \\
10^{-3.5}\end{array}$ & $\mathrm{MS} / \mathrm{RT}$ & Heptanoic acid -d13 & Glycolic acid & $\mathrm{ND}$ & $\mathrm{ND}$ & DMM - 57 & $<\mathrm{QL}$ \\
\hline $\begin{array}{l}\text { EI: } 41,57,73,117,131, \\
147,189,2011,231,, 301\end{array}$ & 316 & 2 & 88 & 2-Buten-1,4-diol & & $\begin{array}{c}0.5 \\
10^{-4.4}\end{array}$ & Standard & Heptanoic acid -d13 & Succinic acid & ND & $\mathrm{ND}$ & 147 & $<\mathrm{QL}$ \\
\hline $\begin{array}{l}\text { EI : } 41,57,73,115,147, \\
189,261,303\end{array}$ & 318 & 2 & 90 & Oxalic acid & & $\begin{array}{l}2 \\
10^{-5.6}\end{array}$ & $\begin{array}{c}\text { Standard } \\
\text { Cahill et al, (2006) }\end{array}$ & Heptanoic acid -d13 & Succinic acid & DMM - 57 & $<\mathrm{QL}$ & 115 & $277 \pm 17$ \\
\hline $\begin{array}{l}\text { EI : } 43,57,73,99,117 \\
133,147,173,189,247 \\
275,317\end{array}$ & 332 & 2 & 104 & $\begin{array}{l}\text { 2-Hydroxy-2- } \\
\text { methylpropanoic acid }\end{array}$ & & $\begin{array}{l}0.75 \\
10^{-5.3}\end{array}$ & $\begin{array}{c}\text { Nist/MS/RT } \\
\text { Rossignol (2012) }\end{array}$ & Heptanoic acid -d13 & Glycolic acid & 147 & $41 \pm 100$ & DMM - 57 & blank \\
\hline $\begin{array}{l}\text { EI : } 41,57,73,75,115, \\
133,147,189,218,303, \\
318\end{array}$ & 318 & 2 & 90 & 3-Hydroxypropanoic acid & & $\begin{array}{c}1 \\
10^{-5.1}\end{array}$ & $\begin{array}{c}\text { Nist/SM/TR } \\
\text { Rossignol (2012) }\end{array}$ & Heptanoic acid -d13 & Glycolic acid & DMM - 57 & $39 \pm 62$ & DMM - 57 & $<\mathrm{QL}$ \\
\hline $\begin{array}{l}\text { EI : } 41,75,117,129,131, \\
171,215,257\end{array}$ & 272 & 1 & 158 & Nonanoic acid & & $\begin{array}{l}0.22 \\
10^{-5.5}\end{array}$ & $\begin{array}{c}\text { MS/RT/Nist } \\
\text { Rossignol (2012) }\end{array}$ & Heptanoic acid -d13 & Heptanoic acid & 131 & blank & DMM - 57 & blank \\
\hline
\end{tabular}


Identification and quantification of compounds during ChArMEx campaign.

DMM : Derivatized Molar Mass ; MM : Molar Mass ; MS : Mass Spectra ; RT : Retention Time ; QL : Quantification Limit ; ND : Not Detected

\begin{tabular}{|c|c|c|c|c|c|c|c|c|c|c|c|c|c|}
\hline \multicolumn{8}{|c|}{ Identification } & \multicolumn{6}{|c|}{ Quantification } \\
\hline \multirow[b]{2}{*}{ Main Fragments } & \multirow[b]{2}{*}{ DMM } & \multirow{2}{*}{$\begin{array}{l}\text { Number } \\
\text { of } \\
\text { functions }\end{array}$} & \multirow[b]{2}{*}{ MM } & \multirow[b]{2}{*}{ Name } & \multirow[b]{2}{*}{ Structure } & \multirow{2}{*}{$\begin{array}{c}\mathrm{O} / \mathrm{C} \\
\begin{array}{c}\log (\text { Saturation Vapor } \\
\text { Pressure) }\end{array}\end{array}$} & \multirow[b]{2}{*}{ Identification method } & \multirow[b]{2}{*}{ Substitut } & \multirow[b]{2}{*}{ External standard } & \multicolumn{2}{|c|}{ Gaseous Phase } & \multicolumn{2}{|c|}{ Particulate phase } \\
\hline & & & & & & & & & & $\begin{array}{c}\mathrm{m} / \mathrm{z} \\
\text { integrated }\end{array}$ & $\begin{array}{c}\text { Concentration } \\
\left(\mathrm{ng} / \mathrm{m}^{3}\right)\end{array}$ & $\begin{array}{c}\mathrm{m} / \mathrm{z} \\
\text { integrated }\end{array}$ & $\begin{array}{c}\text { Concentration } \\
\left(\mathrm{ng} / \mathrm{m}^{3}\right)\end{array}$ \\
\hline $\begin{array}{l}\text { EI: } 41,57,73,115,133, \\
147,189,275,317\end{array}$ & 332 & 2 & 104 & Malonic acid & & $\begin{array}{c}1.3 \\
10^{-6.1}\end{array}$ & $\begin{array}{c}\text { Nist/MS/RT } \\
\text { Cahill et al, (2006) }\end{array}$ & Heptanoic acid - $\mathrm{d} 13$ & Succinic acid & ND & ND & 73 & $3 \pm 1$ \\
\hline $\begin{array}{l}\text { EI : } 45,57,73,133,147, \\
157,185,259,301\end{array}$ & 316 & 2 & 88 & $\begin{array}{l}\text { 2-Hydroxy-2-propenoic } \\
\text { acid }\end{array}$ & & $\begin{array}{c}1 \\
10^{-3.6}\end{array}$ & $\mathrm{MS} / \mathrm{RT}$ & Heptanoic acid - $\mathrm{d} 13$ & Glycolic acid & ND & ND & DMM - 57 & $<\mathrm{QL}$ \\
\hline $\begin{array}{l}\text { EI: }: 41,57,73,133,147, \\
199,273,315\end{array}$ & 330 & 2 & 102 & 3-Hydroxy-2-butenoic acid & & $\begin{array}{l}0.75 \\
10^{-4.1}\end{array}$ & Nist/MS/RT & Heptanoic acid -d13 & Glycolic acid & $\mathrm{ND}$ & $\mathrm{ND}$ & DMM - 57 & $<\mathrm{QL}$ \\
\hline$\underset{271}{\text { EI }: 41,75,129,131,229,}$ & 286 & 1 & 172 & Decanoic acid & & $\begin{array}{c}0.2 \\
10^{-6.0}\end{array}$ & $\begin{array}{c}\text { Nist/MS/RT } \\
\text { Cahill et al, (2006) }\end{array}$ & Heptanoic acid - $\mathrm{d} 13$ & Heptanoic acid & DMM - 57 & blank & DMM - 57 & blank \\
\hline $\begin{array}{l}\text { EI : } 41,57,73,117,133, \\
147,189,287,329\end{array}$ & 344 & 2 & 116 & Maleic acid & & $\begin{array}{c}1 \\
10^{-6.9}\end{array}$ & $\begin{array}{c}\text { Nist/MS/RT } \\
\text { Cahill et al, (2006) }\end{array}$ & Heptanoic acid -d 13 & Succinic acid & $\mathrm{ND}$ & $\mathrm{ND}$ & DMM - 15 & $2 \pm 1$ \\
\hline $\begin{array}{l}\text { EI : } 41,57,73,115,129, \\
147,189,243,289,331\end{array}$ & 346 & 2 & 118 & Succinic acid & & $\begin{array}{c}1 \\
10^{-6.6}\end{array}$ & $\begin{array}{c}\text { Standard } \\
\text { Cahill et al, (2006) }\end{array}$ & Heptanoic acid -d13 & Succinic acid & MMD - 57 & blank & DMM - 131 & $27 \pm 2$ \\
\hline $\begin{array}{l}\text { EI : } 41,73,115,147,189, \\
229,303,345\end{array}$ & 360 & 2 & 132 & A2-Methylsuccinic acid & & $\begin{array}{c}0.8 \\
10^{-7.0}\end{array}$ & $\begin{array}{c}\text { MS/RT } \\
\text { Kawamura et Yasui } \\
(2005)\end{array}$ & Heptanoic acid -d13 & Glutaric acid & $\mathrm{ND}$ & $\mathrm{ND}$ & DMM- 57 & blank \\
\hline $\begin{array}{l}\text { EI }: 41,57,73,75,115, \\
133,147,289,301,343\end{array}$ & 358 & 2 & 130 & $\begin{array}{l}\text { 2-Hydroxy-3-Methyl-2- } \\
\text { pentenoic acid }\end{array}$ & & $\begin{array}{c}0.5 \\
10^{-5.0}\end{array}$ & MS/RT/Nist & Heptanoic acid -d13 & Glutaric acid & ND & ND & DMM - 57 & $4 \pm 1$ \\
\hline $\begin{array}{l}\mathbf{E I}: 41,57,73,75,84,113, \\
133,156,230,245,287, \\
329\end{array}$ & 344 & 2 & 116 & Fumaric acid & & $\begin{array}{c}1 \\
10^{-6.9}\end{array}$ & $\begin{array}{c}\text { MS/RT/Nist } \\
\text { Rossignol (2012) } \\
\text { Cahill et al, (2006) }\end{array}$ & Heptanoic acid -d13 & Succinic acid & ND & $\mathrm{ND}$ & DMM - 15 & $3 \pm 1$ \\
\hline $\begin{array}{l}\text { EI : } 41,57,75,95,129 \\
131,243,285,350\end{array}$ & 300 & 1 & 186 & Undecanoic acid & & $\begin{array}{l}0.18 \\
10^{-6.5}\end{array}$ & $\begin{array}{c}\text { Nist/MS/RT } \\
\text { Cahill et al, (2006) }\end{array}$ & Heptanoic acid -d13 & Heptanoic acid & DMM - 57 & blank & ND & ND \\
\hline $\begin{array}{l}\text { EI: } 41,57,75,83,97,115, \\
129,143,185,227,243, \\
285\end{array}$ & 300 & 1 & 186 & 1-Dodecanol & & $\begin{array}{l}0.08 \\
10^{-5.7}\end{array}$ & MS/RT/Nist & Heptanoic acid -d13 & Heptanoic acid & DMM - 57 & blank & ND & $\mathrm{ND}$ \\
\hline $\begin{array}{l}\text { EI : } 41,57,73,75,129 \\
147,189,303,345\end{array}$ & 360 & 2 & 132 & Glutaric acid & & $\begin{array}{c}0.8 \\
10^{-7.1}\end{array}$ & $\begin{array}{c}\text { Standard } \\
\text { Cahill et al, (2006) }\end{array}$ & Heptanoic acid - $\mathrm{d} 13$ & Glutaric acid & $\mathrm{ND}$ & ND & DMM - 57 & $16 \pm 1$ \\
\hline $\begin{array}{l}\text { EI: } 41,57,73,89,115, \\
133,147,171,189,245, \\
377,419\end{array}$ & 434 & 3 & 92 & Glycerol & & $\begin{array}{c}1 \\
10^{-6.3}\end{array}$ & $\begin{array}{c}\text { Standard } \\
\text { Jaoui et al. (2014) }\end{array}$ & Glycerol-d8 & Glycerol & DMM - 57 & $1646 \pm 131$ & DMM - 57 & $56 \pm 4$ \\
\hline $\begin{array}{l}\text { EI: } 73,75,147,189,221, \\
257,295,323,365\end{array}$ & 380 & 2 & 152 & Mandelic acid & & $\begin{array}{l}0.38 \\
10^{-7.8}\end{array}$ & $\mathrm{MS} / \mathrm{RT}$ & Heptanoic acid -d13 & Glycolic acid & DMM - 57 & $21 \pm 1$ & $\mathrm{ND}$ & $\mathrm{ND}$ \\
\hline
\end{tabular}


Identification and quantification of compounds during ChArMEx campaign.

DMM : Derivatized Molar Mass ; MM : Molar Mass ; MS : Mass Spectra ; RT : Retention Time ; QL : Quantification Limit ; ND : Not Detected

\begin{tabular}{|c|c|c|c|c|c|c|c|c|c|c|c|c|c|}
\hline \multicolumn{8}{|c|}{ Identification } & \multicolumn{6}{|c|}{ Quantification } \\
\hline \multirow[b]{2}{*}{ Main Fragments } & \multirow[b]{2}{*}{ DMM } & \multirow{2}{*}{$\begin{array}{l}\text { Number } \\
\text { of } \\
\text { functions }\end{array}$} & \multirow[b]{2}{*}{ MM } & \multirow[b]{2}{*}{ Name } & \multirow[b]{2}{*}{ Structure } & \multirow{2}{*}{$\begin{array}{c}\text { O/C } \\
\text { Log (Saturation Vapor } \\
\text { Pressure) }\end{array}$} & \multirow[b]{2}{*}{ Identification method } & \multirow[b]{2}{*}{ Substitut } & \multirow[b]{2}{*}{ External standard } & \multicolumn{2}{|c|}{ Gaseous Phase } & \multicolumn{2}{|c|}{ Particulate phase } \\
\hline & & & & & & & & & & $\begin{array}{c}\mathrm{m} / \mathrm{z} \\
\text { integrated }\end{array}$ & $\begin{array}{c}\text { Concentration } \\
\left(\mathrm{ng} / \mathrm{m}^{3}\right)\end{array}$ & $\begin{array}{c}\mathrm{m} / \mathrm{z} \\
\text { integrated }\end{array}$ & $\begin{array}{c}\text { Concentration } \\
\left(\mathrm{ng} / \mathrm{m}^{3}\right)\end{array}$ \\
\hline $\begin{array}{l}\text { EE: } 39,75,89,129,131 \\
257,299\end{array}$ & 314 & 1 & 200 & Dodecanoic acid & & $\begin{array}{l}0.17 \\
10^{-7.1}\end{array}$ & $\begin{array}{c}\text { MS/RT } \\
\text { Rossignol (2012) } \\
\text { Cahill et al, (2006) }\end{array}$ & Heptanoic acid -d13 & Heptanoic acid & DMM - 57 & blank & DMM - 57 & blank \\
\hline $\begin{array}{l}\text { EI: } 41,57,73,133,147, \\
159,175,177,189,221, \\
231,391,433\end{array}$ & 448 & 3 & 106 & $\begin{array}{l}\text { 2,3-Dihydroxypropanoic } \\
\text { acid }\end{array}$ & & $\begin{array}{l}1.3 \\
10^{-7.6}\end{array}$ & $\begin{array}{c}\text { Nist/MS/RT } \\
\text { Rossignol (2012) } \\
\text { (Kourtchev et al., 2008b) }\end{array}$ & $\begin{array}{l}\text { Pentanoic-d9 acid } \\
\text { Glycerol-d8 }\end{array}$ & Glycerol & $\mathrm{ND}$ & ND & DMM - 57 & $20 \pm 2$ \\
\hline $\begin{array}{l}\text { EI }: 41,57,73,111,147, \\
227,309,317,343,359\end{array}$ & 374 & 2 & 146 & Hexanedioic acid & & $\begin{array}{l}0.67 \\
10^{-7.6}\end{array}$ & $\begin{array}{c}\text { MS/RT/Nist } \\
\text { Rossignol (2012) } \\
\text { Cahill et al, (2006) }\end{array}$ & Heptanoic acid -d13 & Succinic acid & ND & ND & DMM - 15 & $1 \pm 1$ \\
\hline $\begin{array}{l}\text { EI : } 41,57,73,115,133, \\
147,189,233,245,273, \\
303,318,377,405,447\end{array}$ & 462 & 3 & 120 & $\begin{array}{l}\text { 2-Methylglyceric acid } \\
\text { (= 2-MGA) }\end{array}$ & & $\begin{array}{c}1 \\
10^{-7.9}\end{array}$ & $\begin{array}{c}\text { Standard } \\
\text { Claeys et al. (2004) }\end{array}$ & $\begin{array}{l}\text { Pentanoic-d9 acid } \\
\text { Glycerol-d8 }\end{array}$ & Glycerol & ND & ND & DMM - 57 & $9 \pm 1$ \\
\hline $\begin{array}{l}\text { EI : } 41,73,133,147,185, \\
197,271,315,343\end{array}$ & 372 & 2 & 144 & $\begin{array}{l}\text { 3-Methyl-2-pentenedioic } \\
\text { acid }\end{array}$ & & $\begin{array}{l}0.67 \\
10^{-7.7}\end{array}$ & MS/RT/Nist & Heptanoic acid -d13 & Glutaric acid & ND & $\mathrm{ND}$ & DMM - 57 & $30 \pm 2$ \\
\hline $\begin{array}{l}\text { EI : } 41,57,73,115,133, \\
147,189,207,221,263, \\
271,377,405,447\end{array}$ & 462 & 3 & 120 & $\begin{array}{l}\text { Erythrose } \\
\text { or Erythrulose }\end{array}$ & & $\begin{array}{c}1.0 \\
10^{-8.0}\end{array}$ & $\begin{array}{c}\text { MS/RT } \\
\text { Jaoui et al. (2014) }\end{array}$ & Pentanoic-d9 acid & 2-MGA & $\mathrm{ND}$ & ND & DMM - 57 & $45 \pm 3$ \\
\hline $\begin{array}{l}\text { EI: } 41,75,129,178,206, \\
271\end{array}$ & 328 & 1 & 214 & Tridecanoic acid & & $\begin{array}{l}0.15 \\
10^{-7.6}\end{array}$ & $\begin{array}{c}\text { MS/RR } \\
\text { Rossignol (2012) } \\
\text { Cahill et al, (2006) }\end{array}$ & Heptanoic acid -d13 & Heptanoic acid & DMM - 57 & $65 \pm 68$ & ND & ND \\
\hline $\begin{array}{l}\text { EI : } 41,57,73,115,133, \\
147,175,185,189,213, \\
287,317,345,391,419 \\
461\end{array}$ & 476 & 3 & 134 & Methyltartronic acid & & $\begin{array}{l}1.25 \\
10^{-9.2}\end{array}$ & $\mathrm{MS} / \mathrm{RT}$ & Heptanoic acid -d13 & Succinic acid & ND & ND & DMM - 131 & $6 \pm 1$ \\
\hline $\begin{array}{l}\text { EI: } 41,57,73,75,111, \\
147,185,317,345,387\end{array}$ & 402 & 2 & 174 & Ketonorlimonic acid & & $\begin{array}{l}0.71 \\
10^{-9.4}\end{array}$ & $\begin{array}{c}\text { MS/RT } \\
\text { Rossignol (2012) } \\
\text { Kleindienst et al. (2007) }\end{array}$ & Heptanoic acid -d13 & Glutaric acid & ND & $\mathrm{ND}$ & DMM - 57 & $15 \pm 1$ \\
\hline $\begin{array}{l}\text { EI : 41, 69, 96, 75, 129, } \\
207,285\end{array}$ & 342 & 1 & 228 & Tetradecanoic acid & & $\begin{array}{l}0.14 \\
10^{-8.0}\end{array}$ & $\begin{array}{c}\text { Nist/MS/RT } \\
\text { Cahill et al, (2006) }\end{array}$ & Heptanoic acid -d13 & Heptanoic acid & DMM - 57 & $314 \pm 100$ & DMM - 57 & blank \\
\hline $\begin{array}{l}\text { EI : } 41,73,115,133,147, \\
189,217,247,287,419, \\
461\end{array}$ & 476 & 3 & 134 & Malic acid & & $\begin{array}{l}1.25 \\
10^{-9.4}\end{array}$ & $\begin{array}{c}\text { Standard } \\
\text { Rossignol (2012) } \\
\text { Cahill et al, (2006) }\end{array}$ & Heptanoic acid -d13 & Glycolic acid & ND & ND & DMM -15 & $33 \pm 3$ \\
\hline $\begin{array}{l}\text { EI : } 41,73,115,147,189, \\
217,273,301,331,405, \\
433,475\end{array}$ & 490 & 3 & 148 & $\begin{array}{c}\text { DHOPA } \\
\left(\begin{array}{l}\text { (4-Xoxo-2,3- } \\
\text { dihydroxypentanoic acid })\end{array}\right.\end{array}$ & & $\begin{array}{c}1.0 \\
10^{-9.3}\end{array}$ & $\begin{array}{l}\text { Standard } \\
\text { Kleindienst et al. (2004) }\end{array}$ & Tartric acid-d2 & Glutaric acid & ND & ND & DMM -57 & $2 \pm 0,1$ \\
\hline $\begin{array}{l}\text { EI : } 41,57,73,105,119 \\
133,147,163,189,221 \\
263,337,399,379\end{array}$ & 394 & 2 & 166 & $\begin{array}{l}\text { 1,2-Benzenedicarboxylic } \\
\text { acid }\end{array}$ & & $\begin{array}{c}0.5 \\
10^{-9.3}\end{array}$ & $\begin{array}{l}\text { Nist/MS/RT } \\
\text { Kleindienst et al. (2012) }\end{array}$ & Heptanoic acid -d13 & Succinic acid & ND & ND & DMM - 131 & $4 \pm 1$ \\
\hline
\end{tabular}


Identification and quantification of compounds during ChArMEx campaign.

DMM : Derivatized Molar Mass ; MM : Molar Mass ; MS : Mass Spectra ; RT : Retention Time ; QL : Quantification Limit ; ND : Not Detected

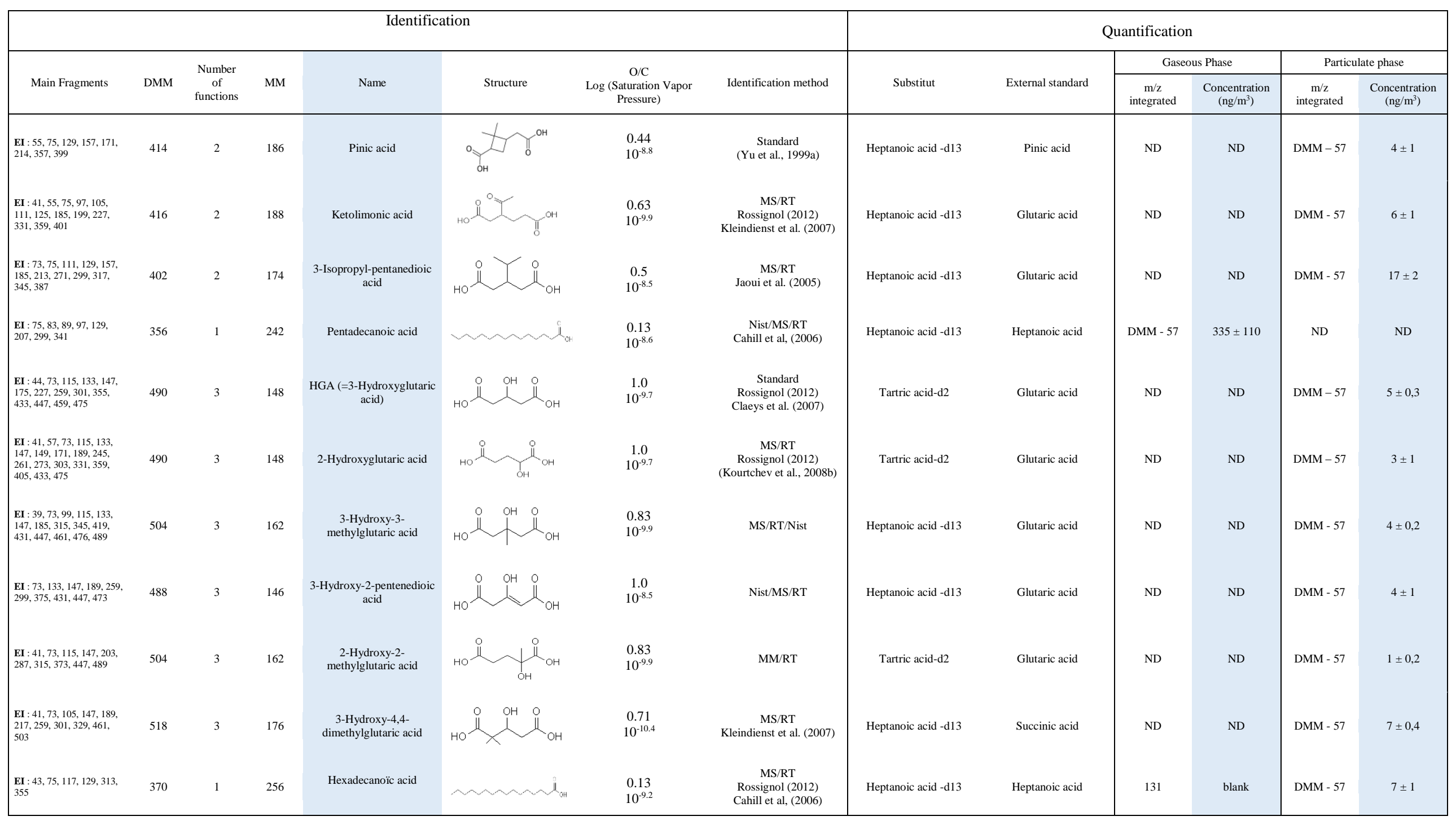


Identification and quantification of compounds during ChArMEx campaign.

DMM : Derivatized Molar Mass ; MM : Molar Mass ; MS : Mass Spectra ; RT : Retention Time ; QL : Quantification Limit ; ND : Not Detected

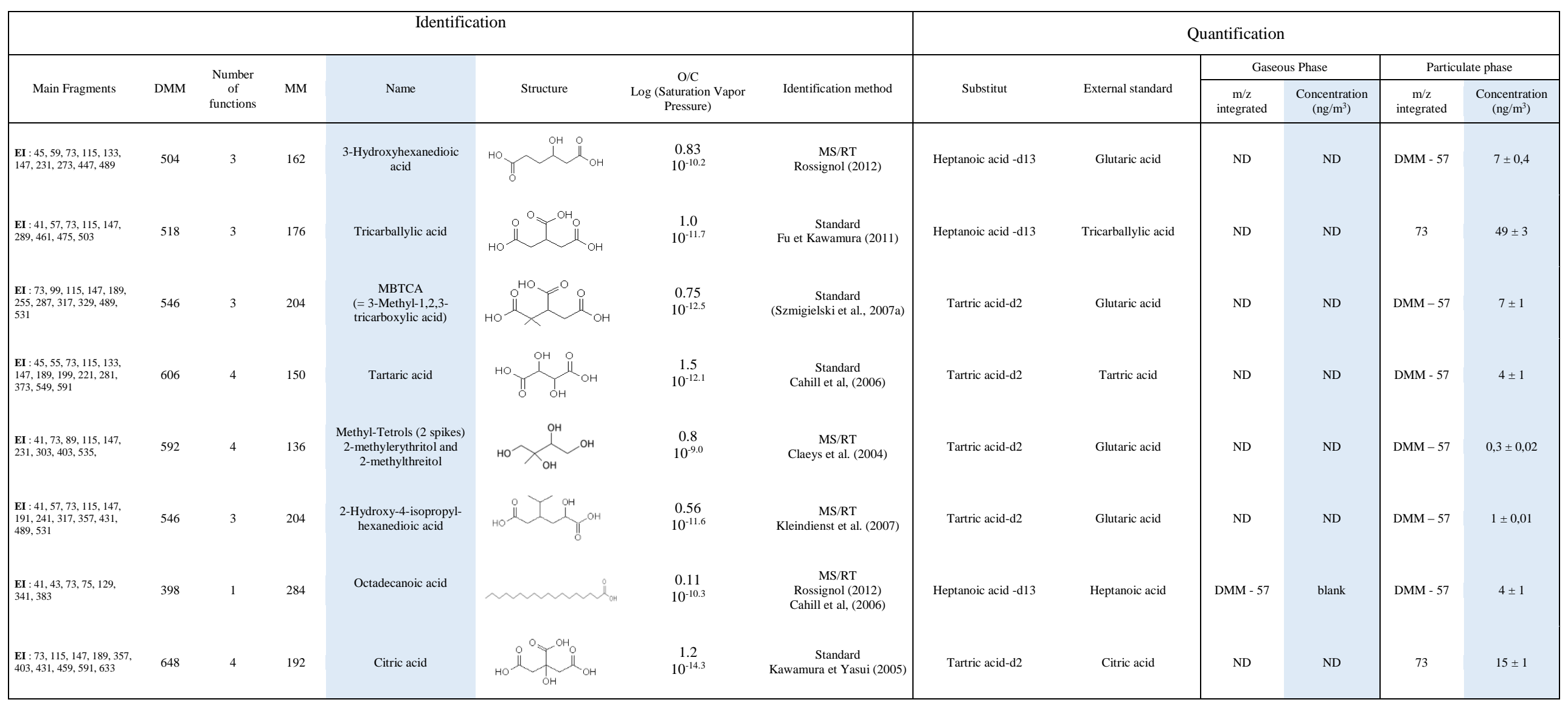


Supplementary material 2: Time series of compounds measured during ChArMEx campaign (in the gas phase in red and in the particulate phase in blue).
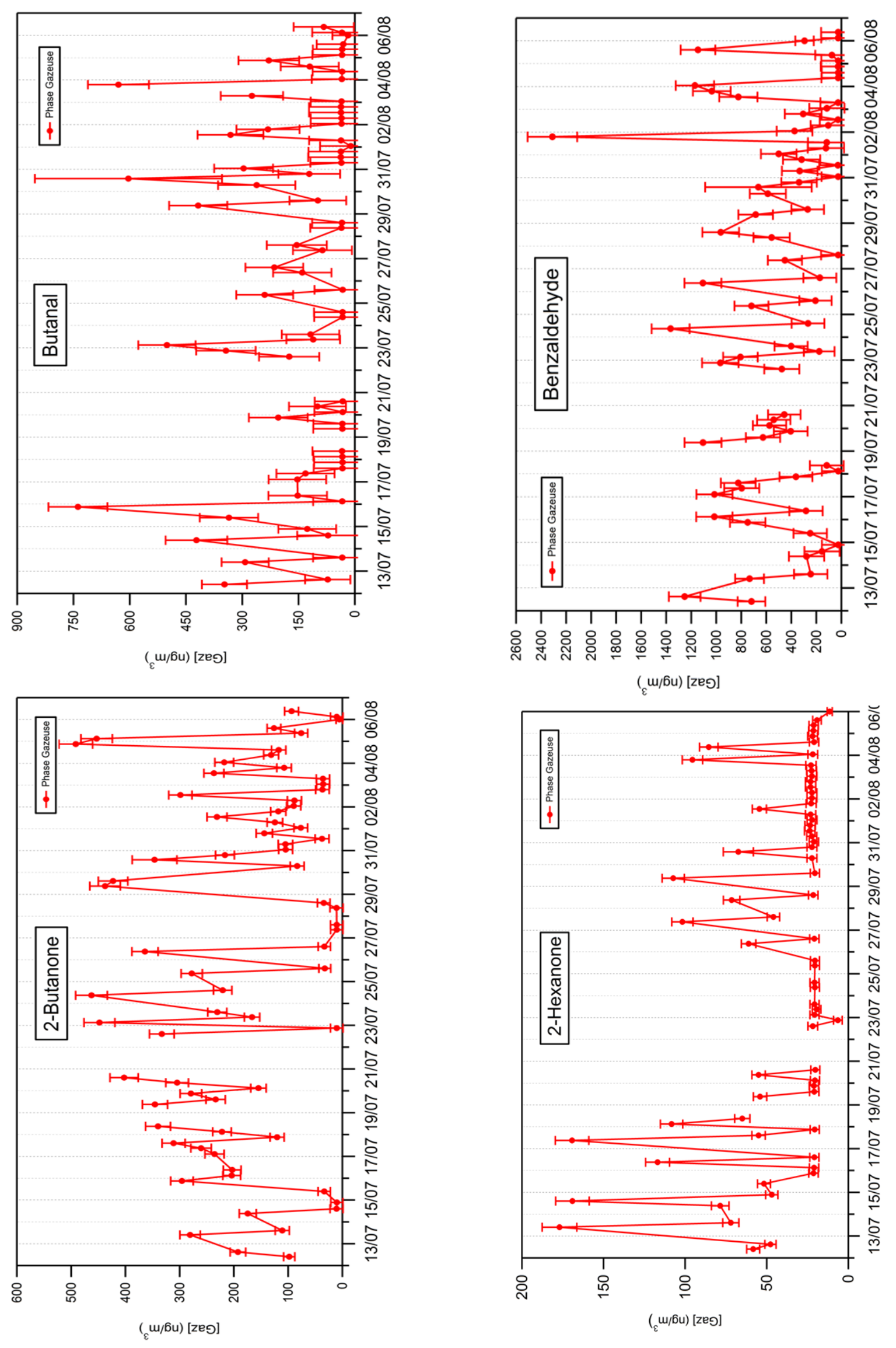

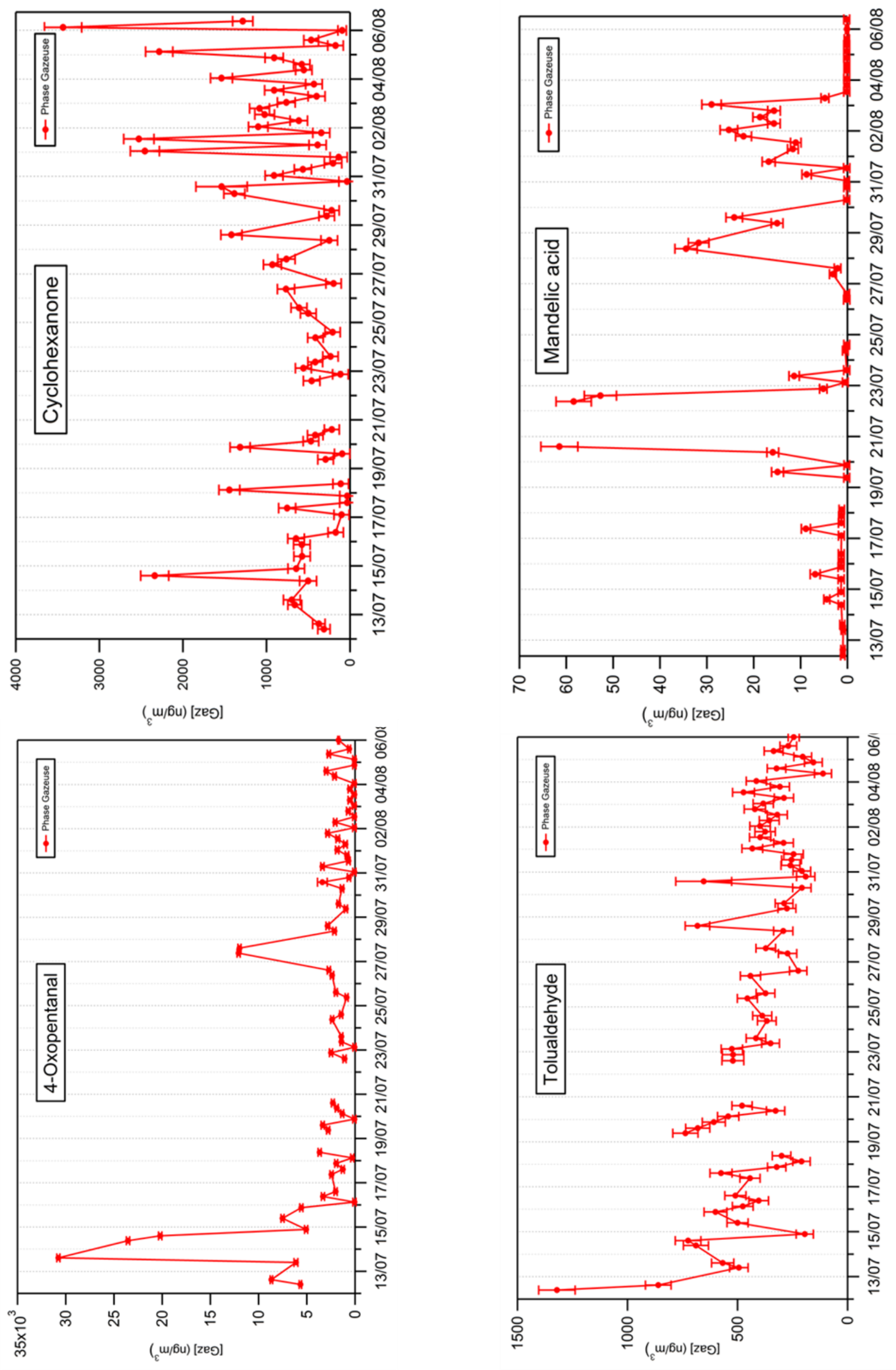

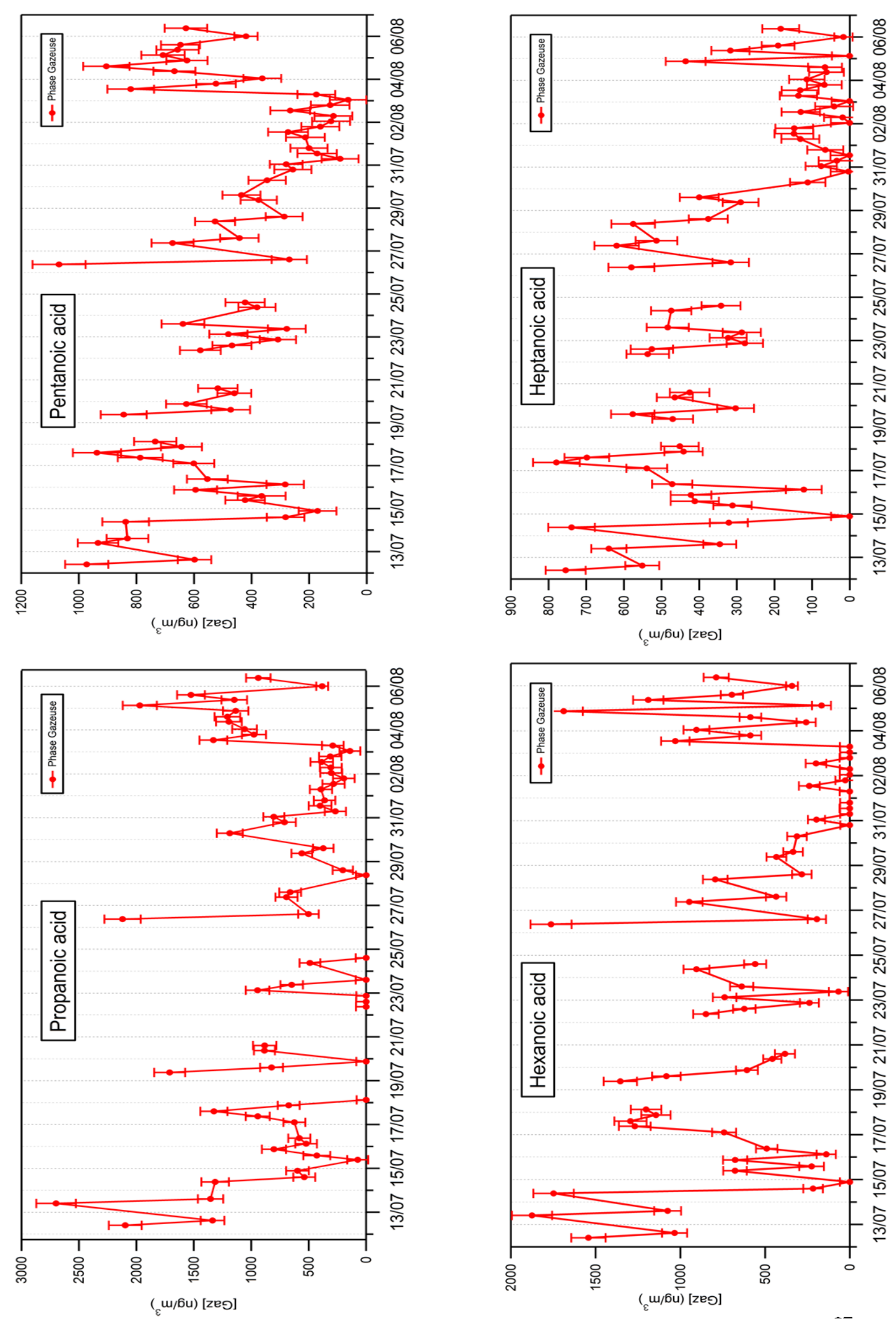

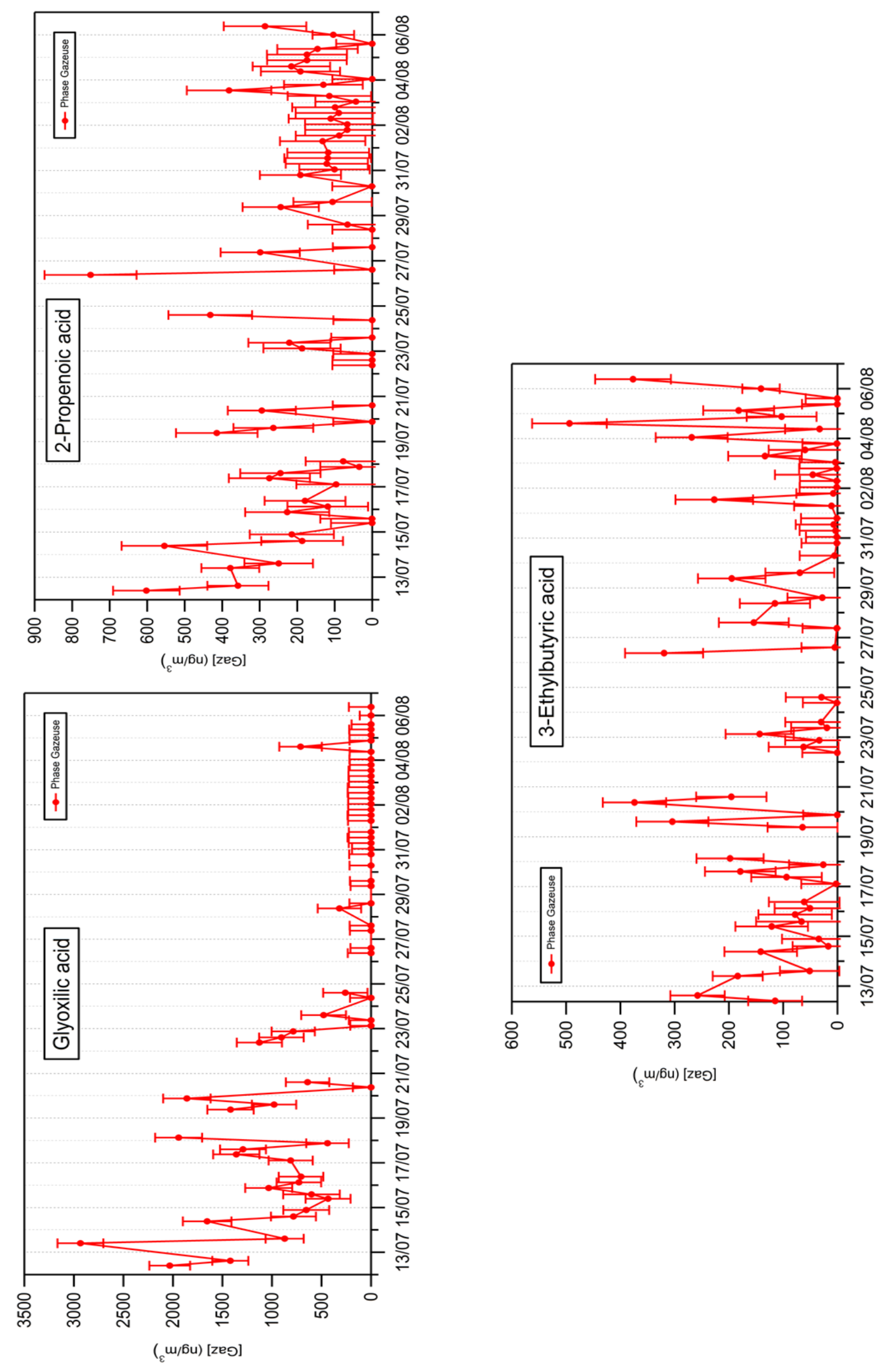

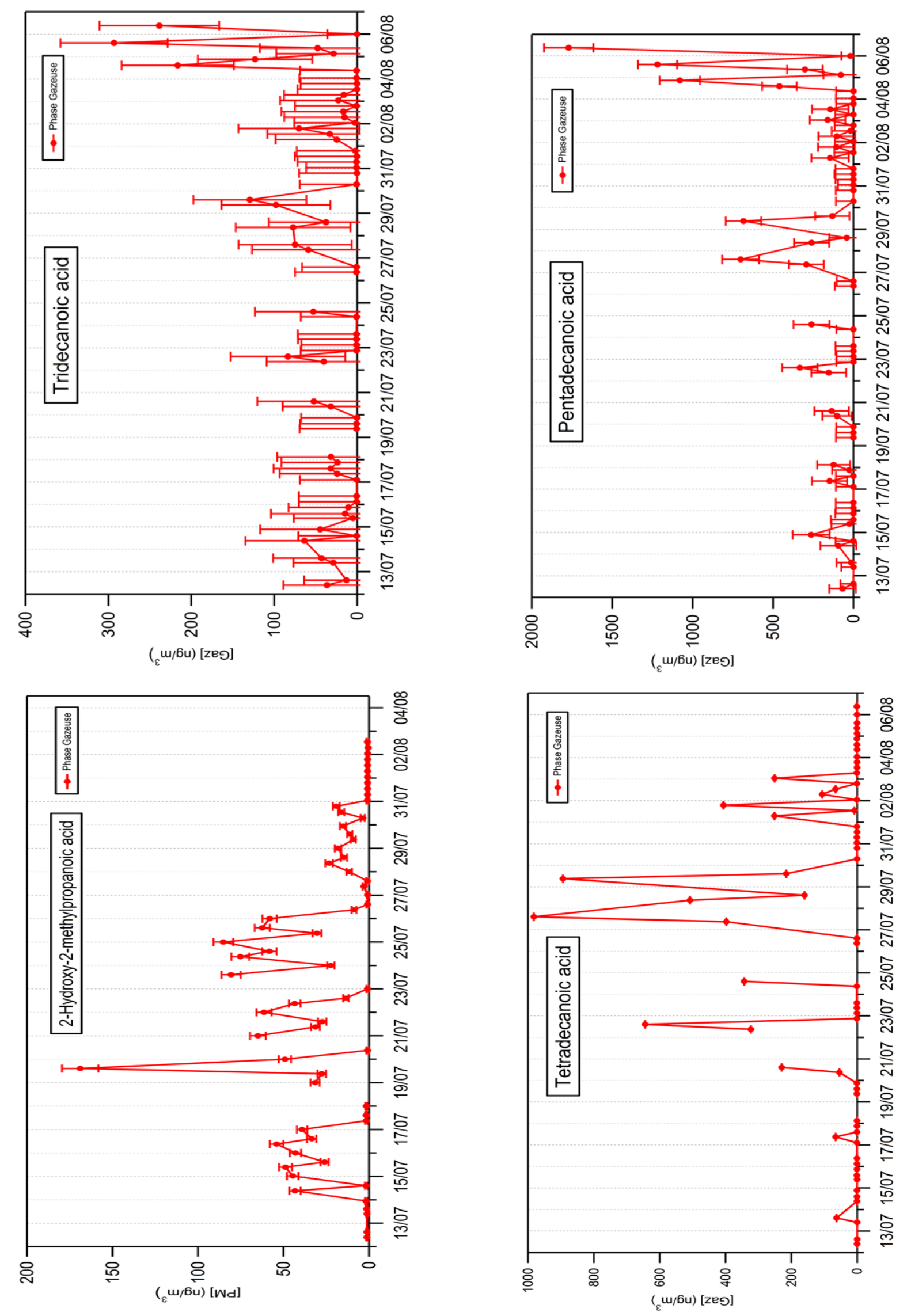

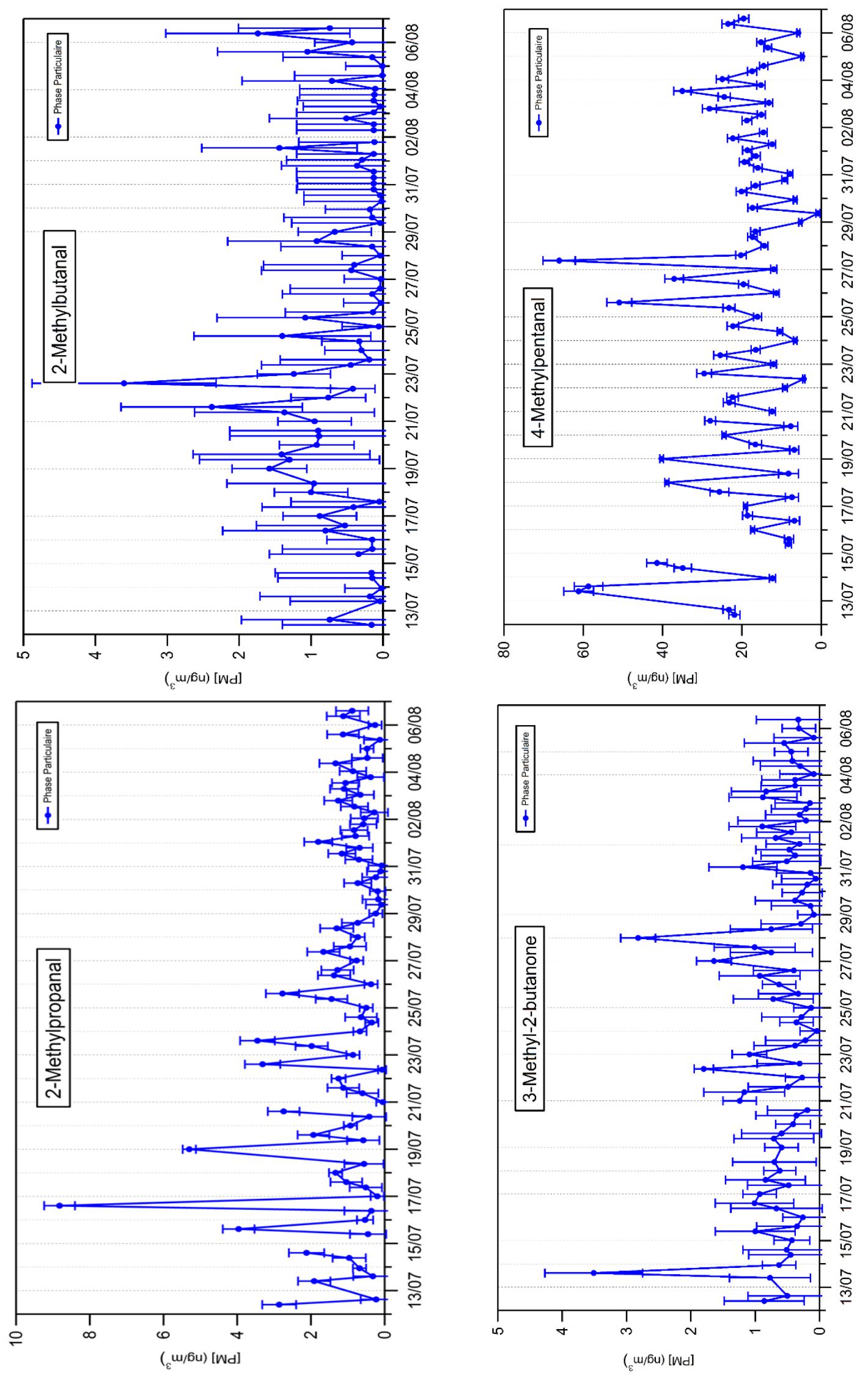

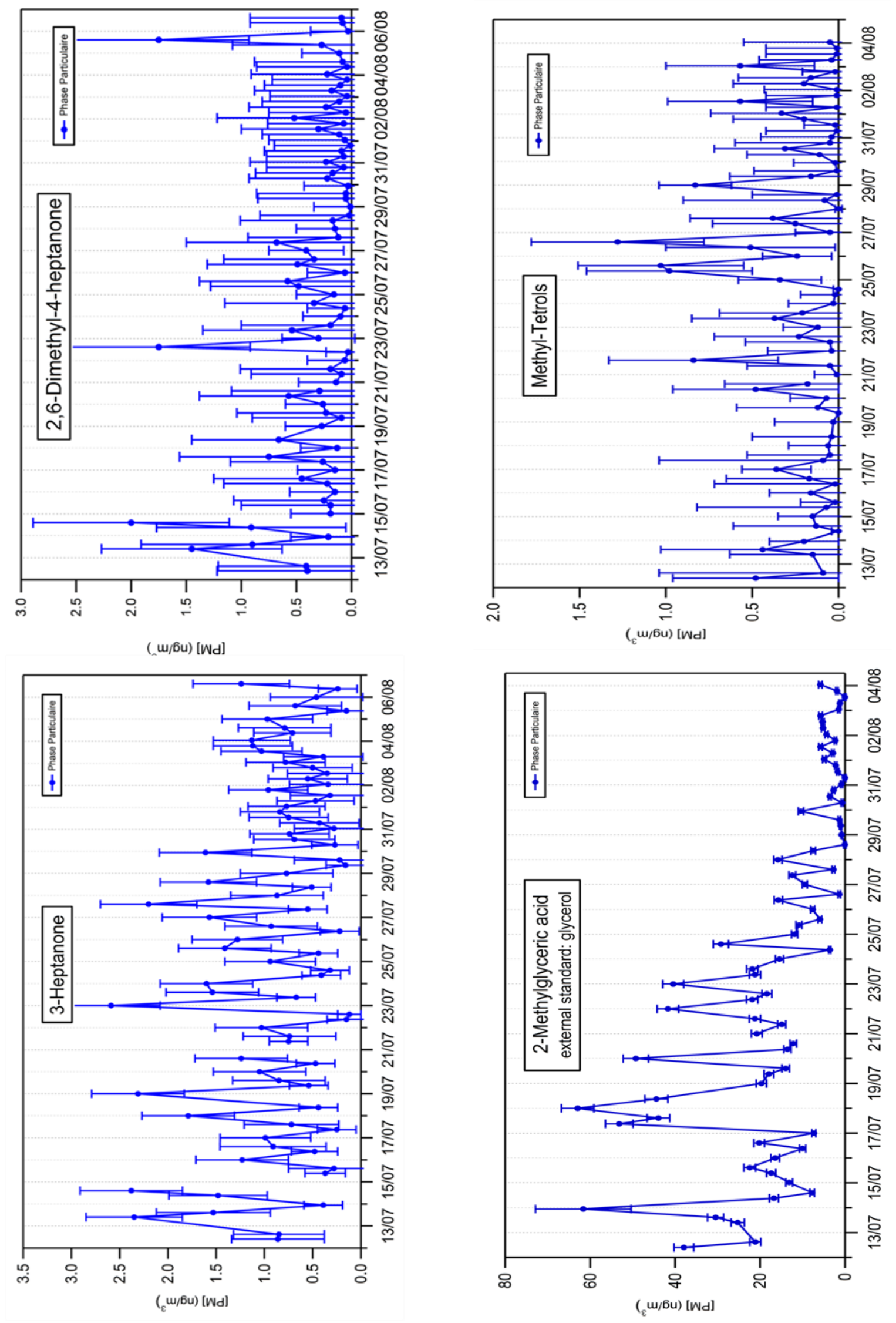

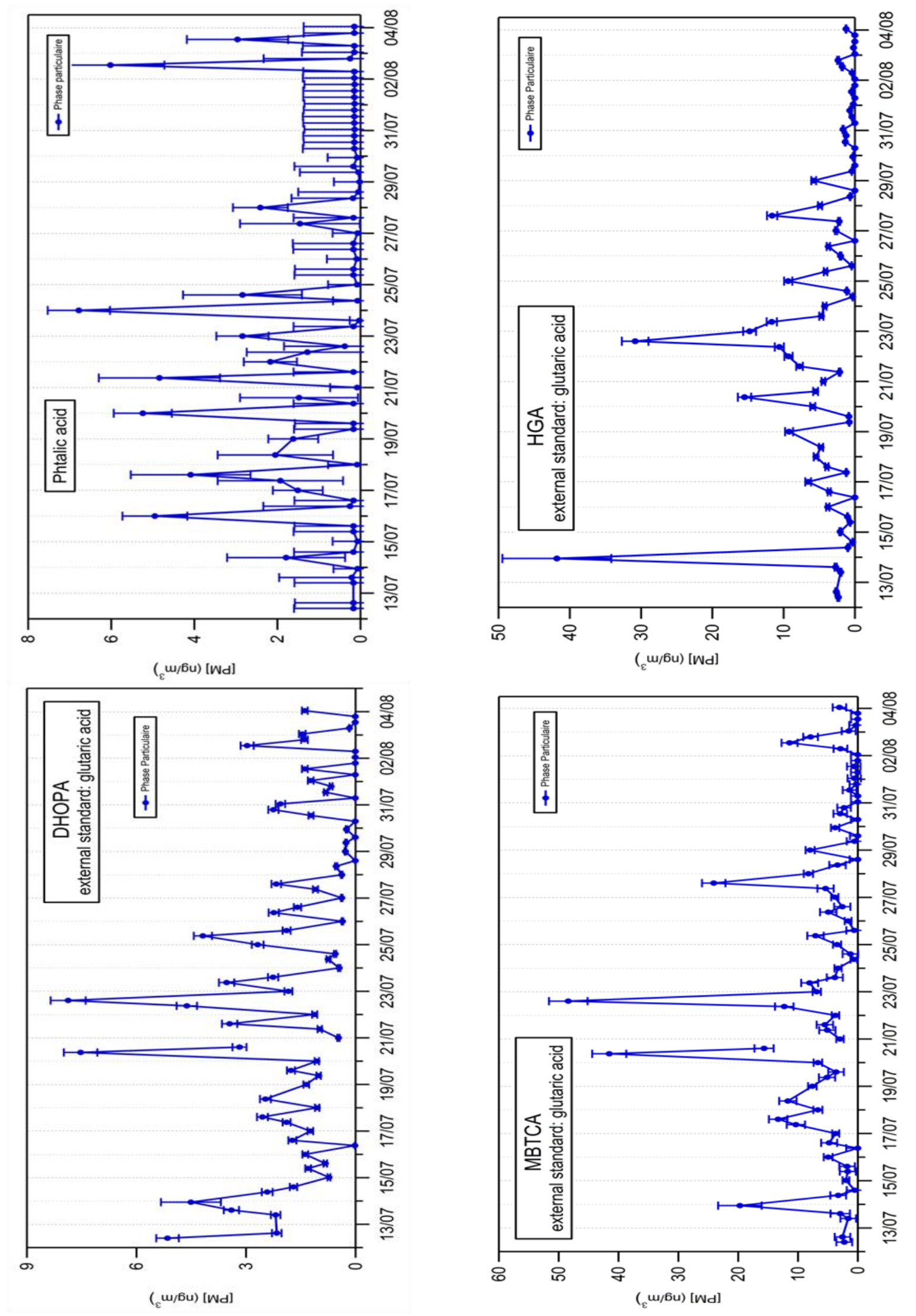

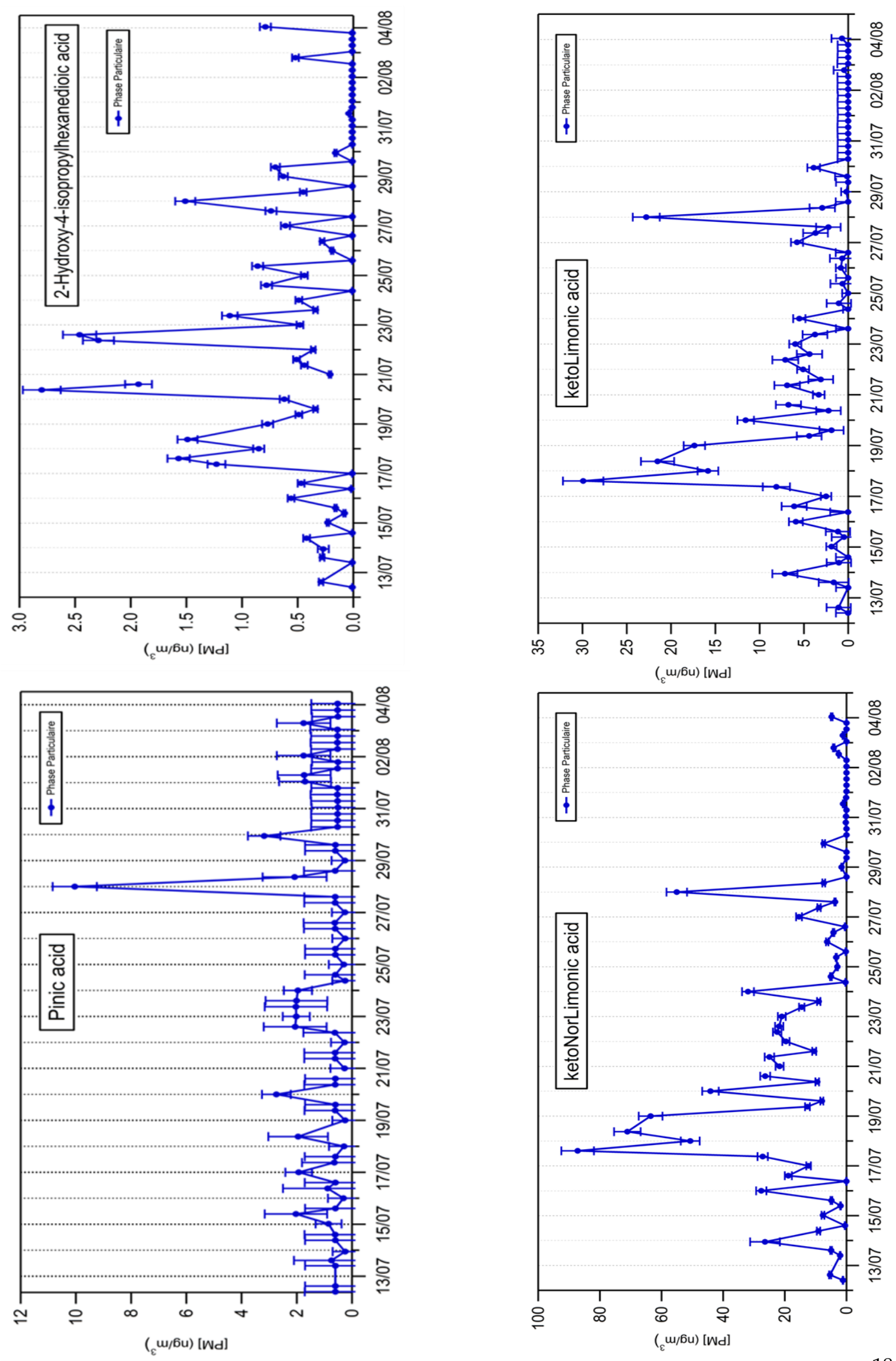

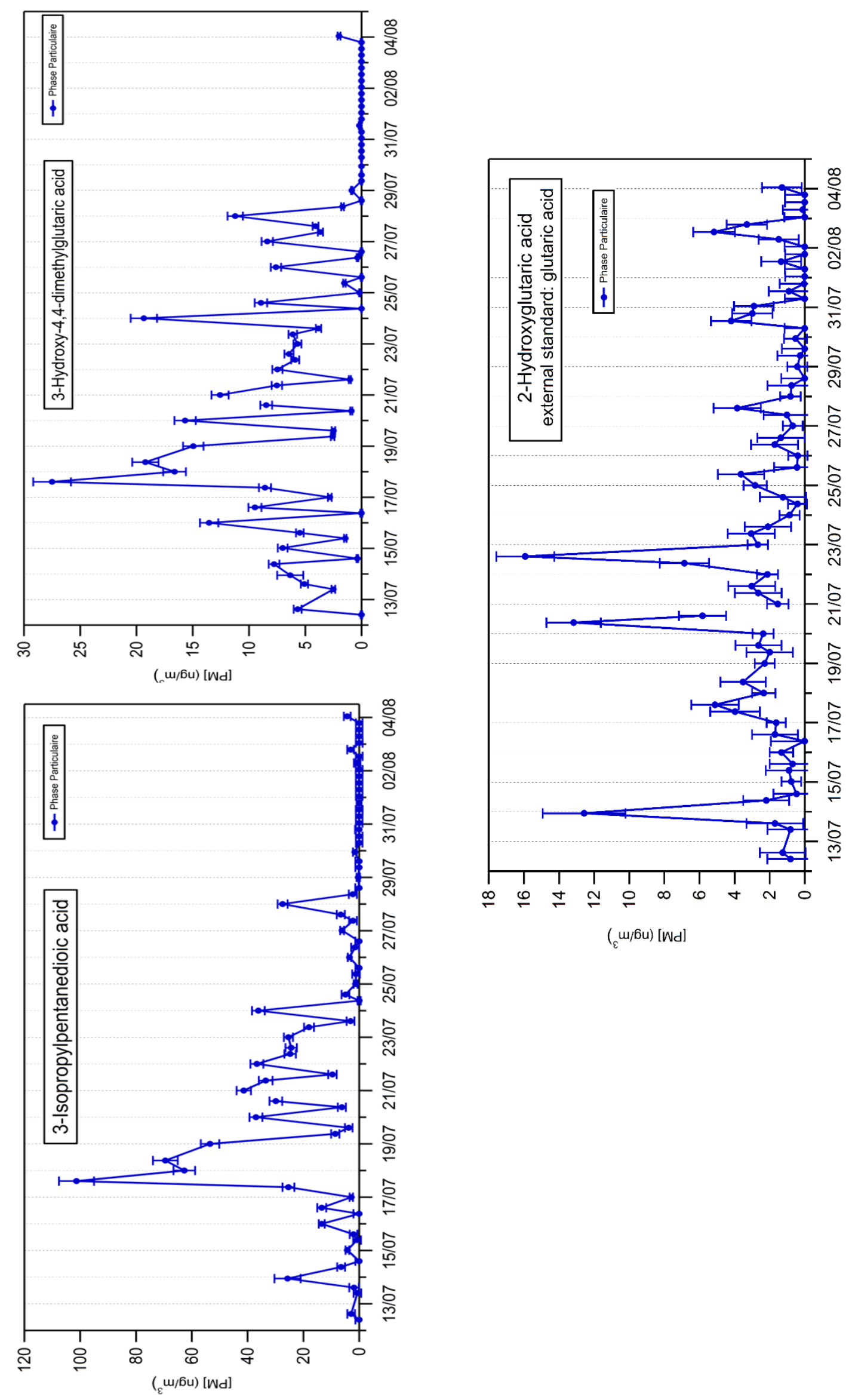

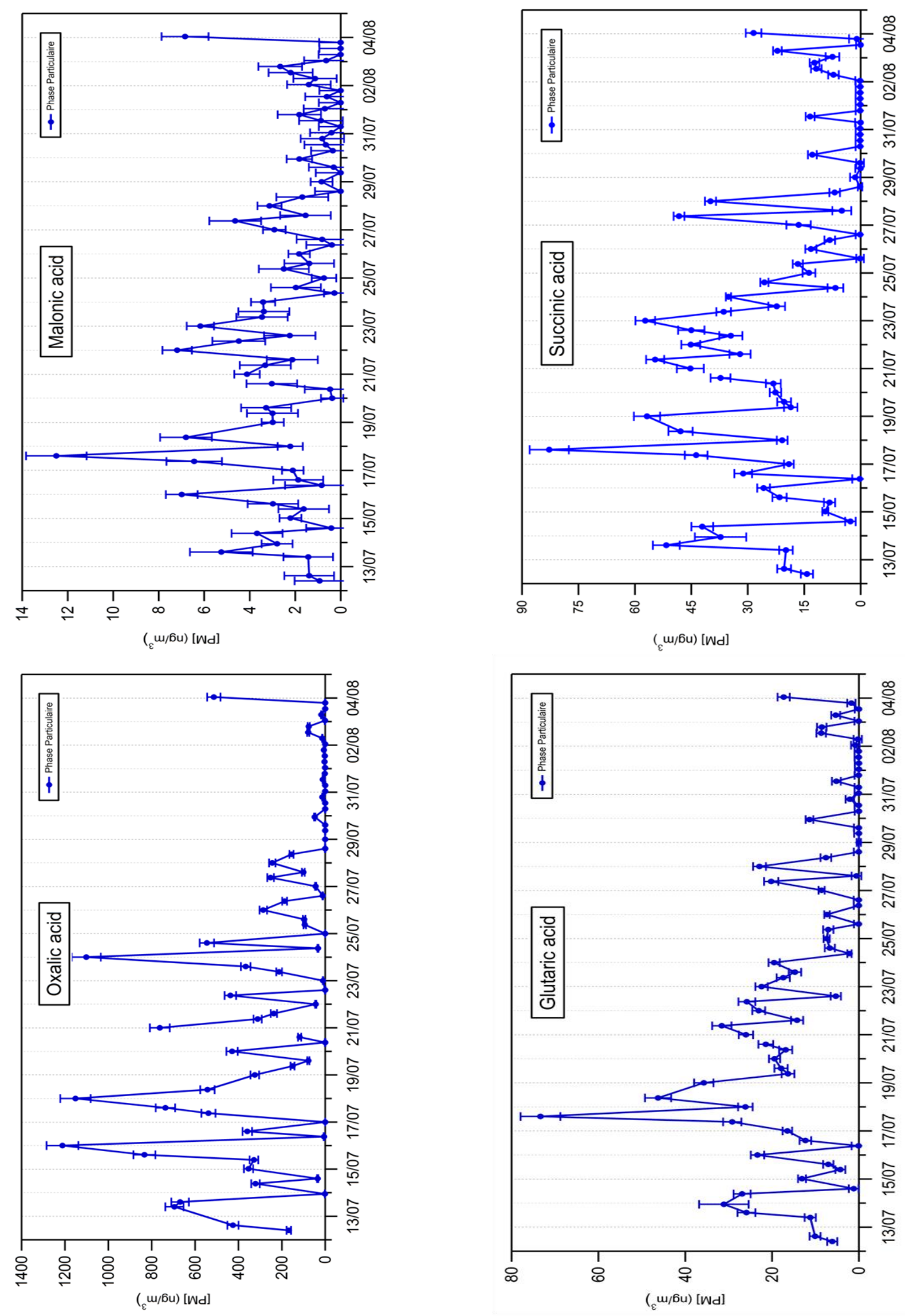

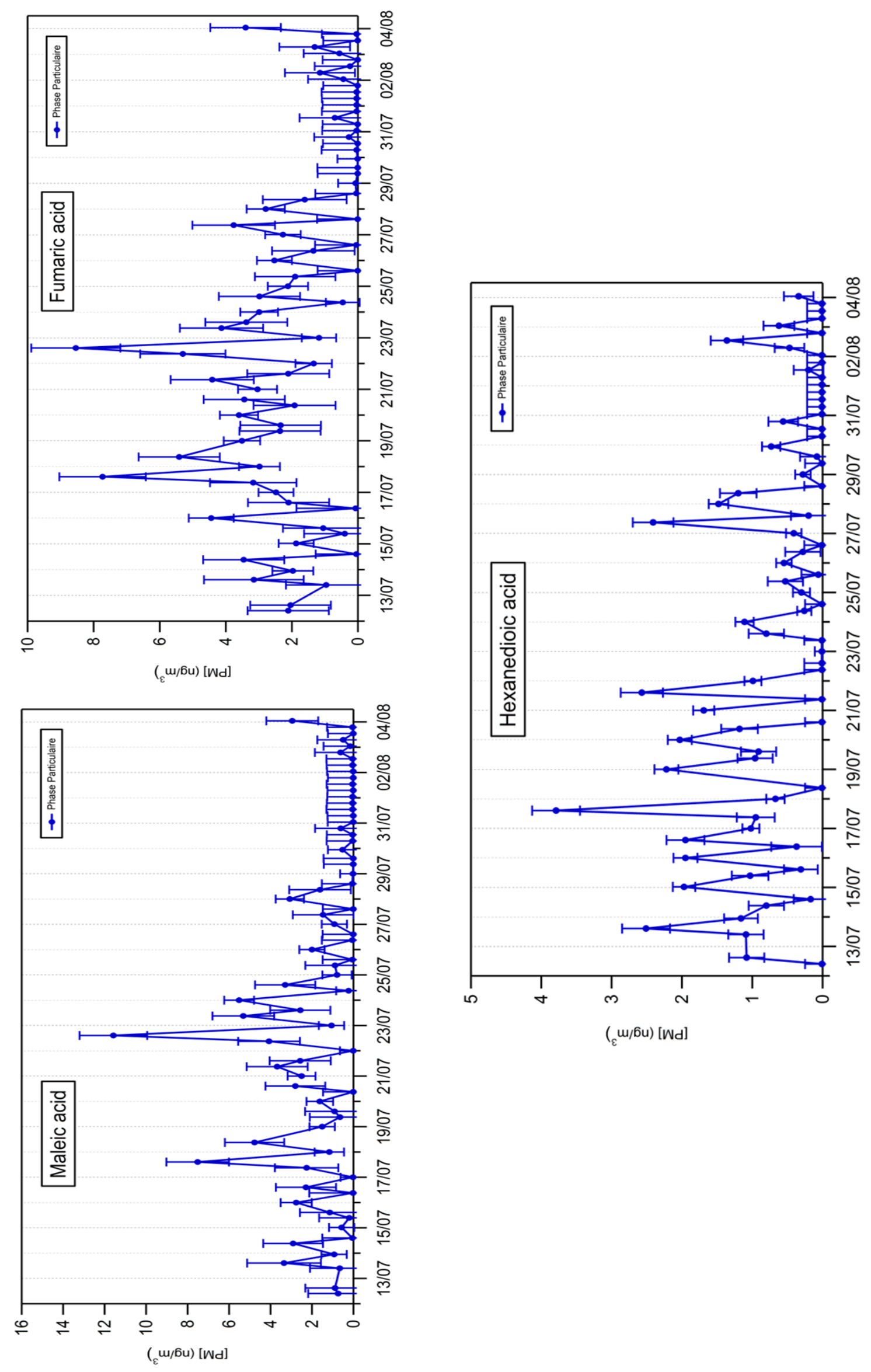

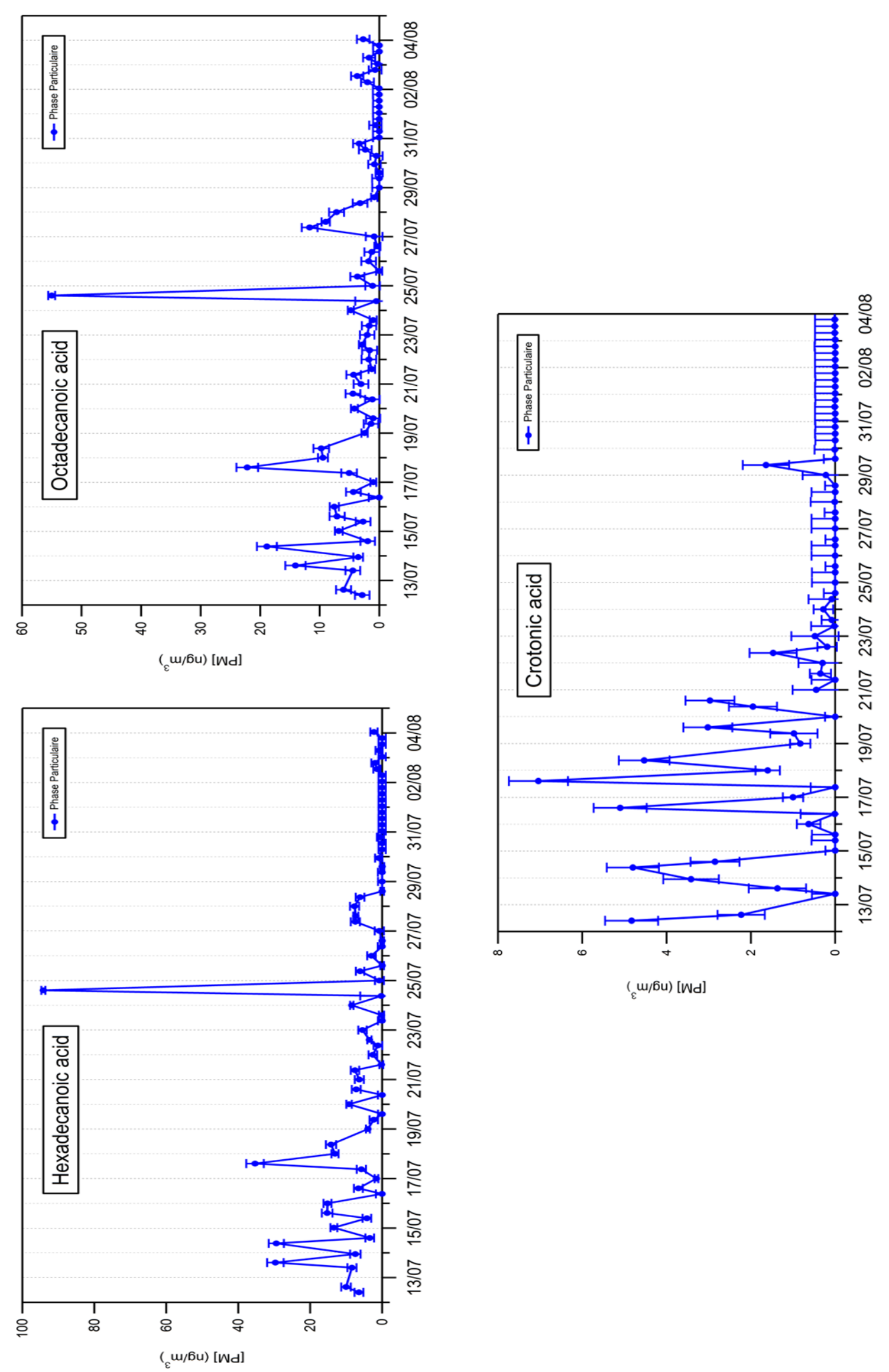


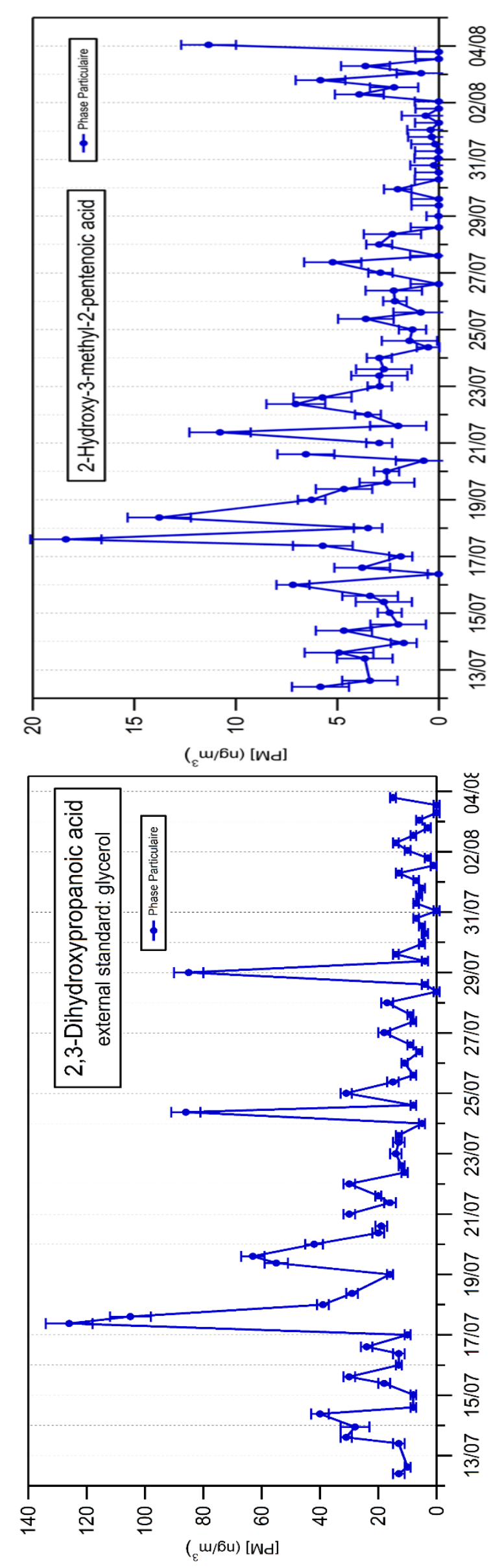



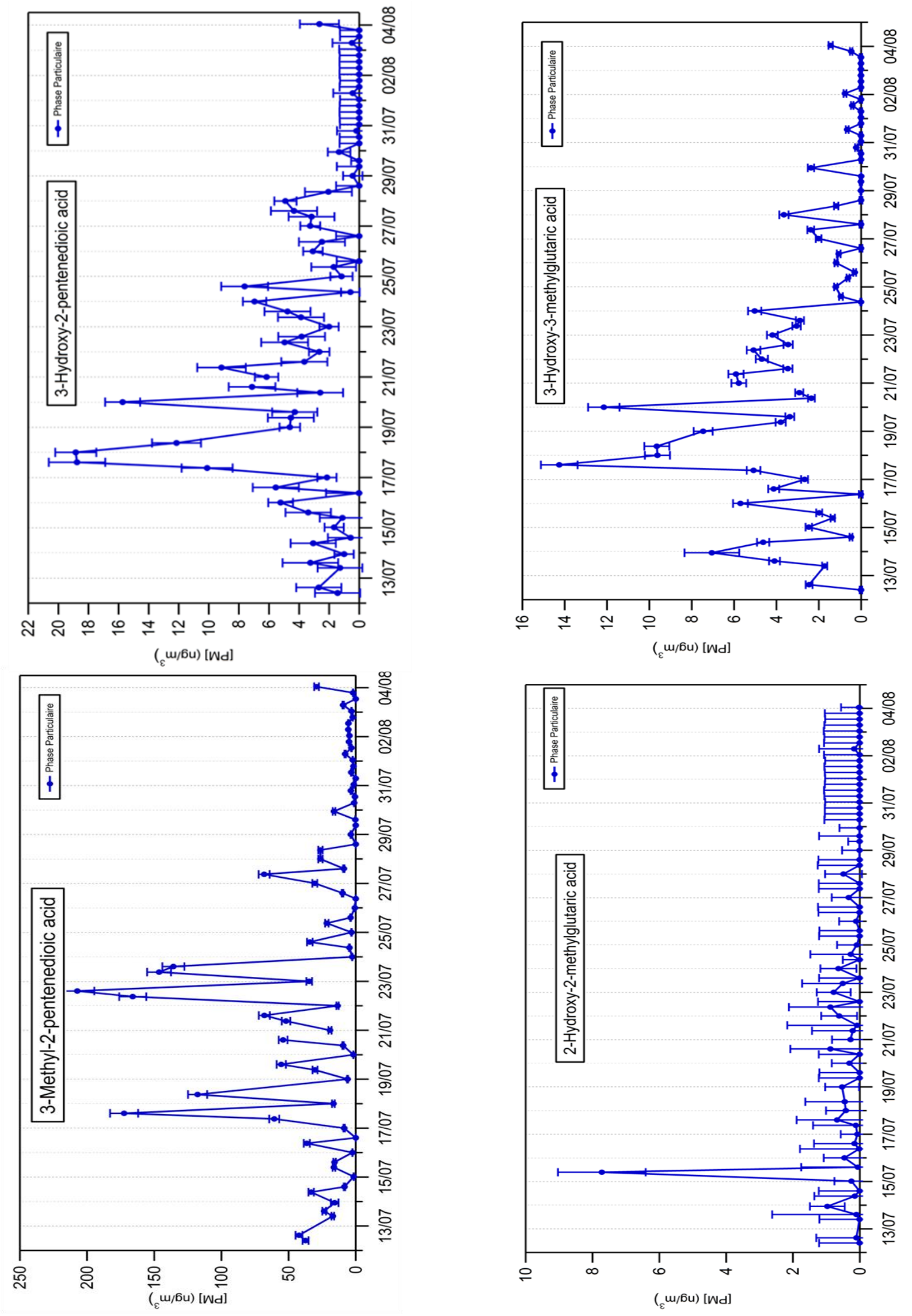

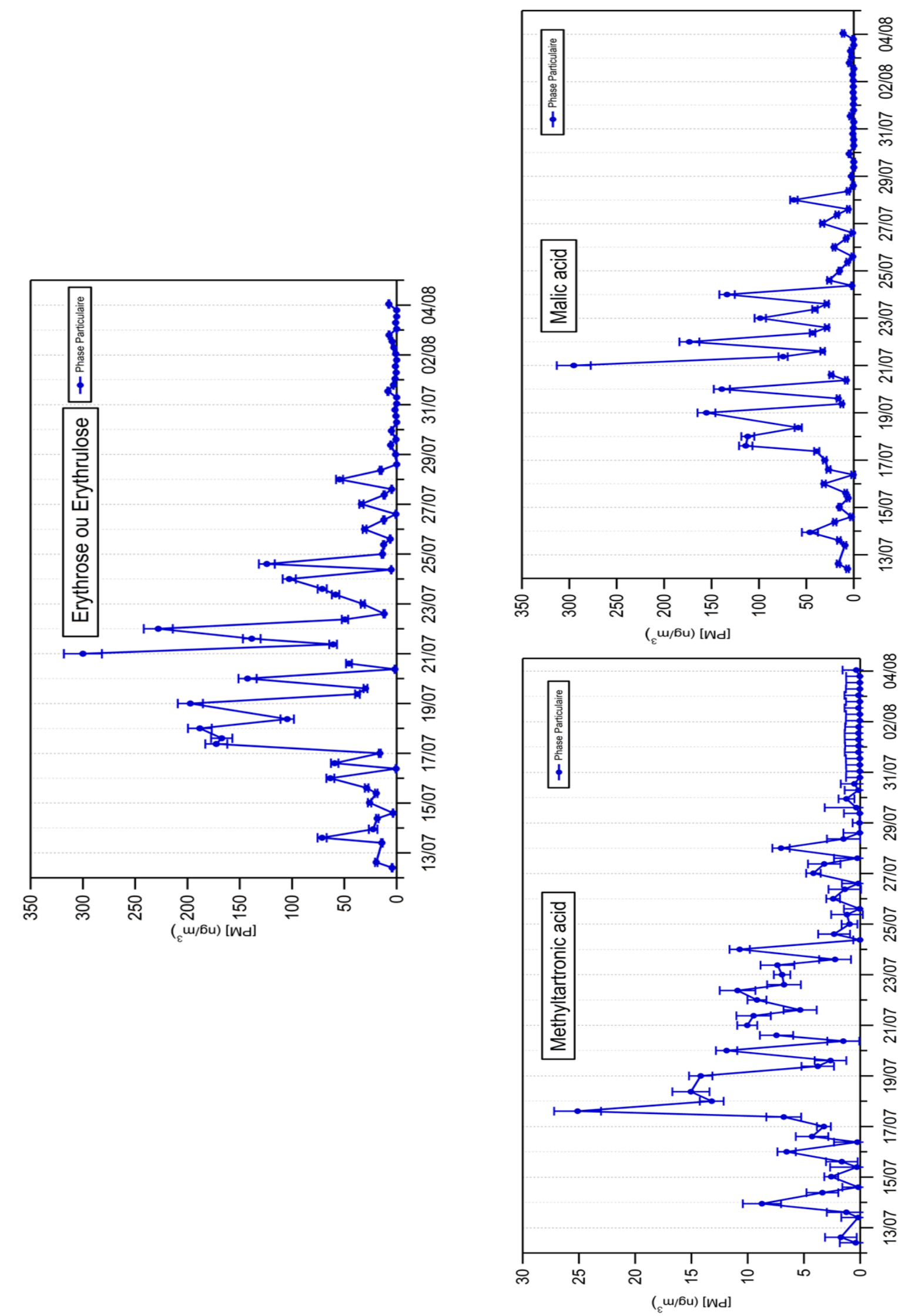

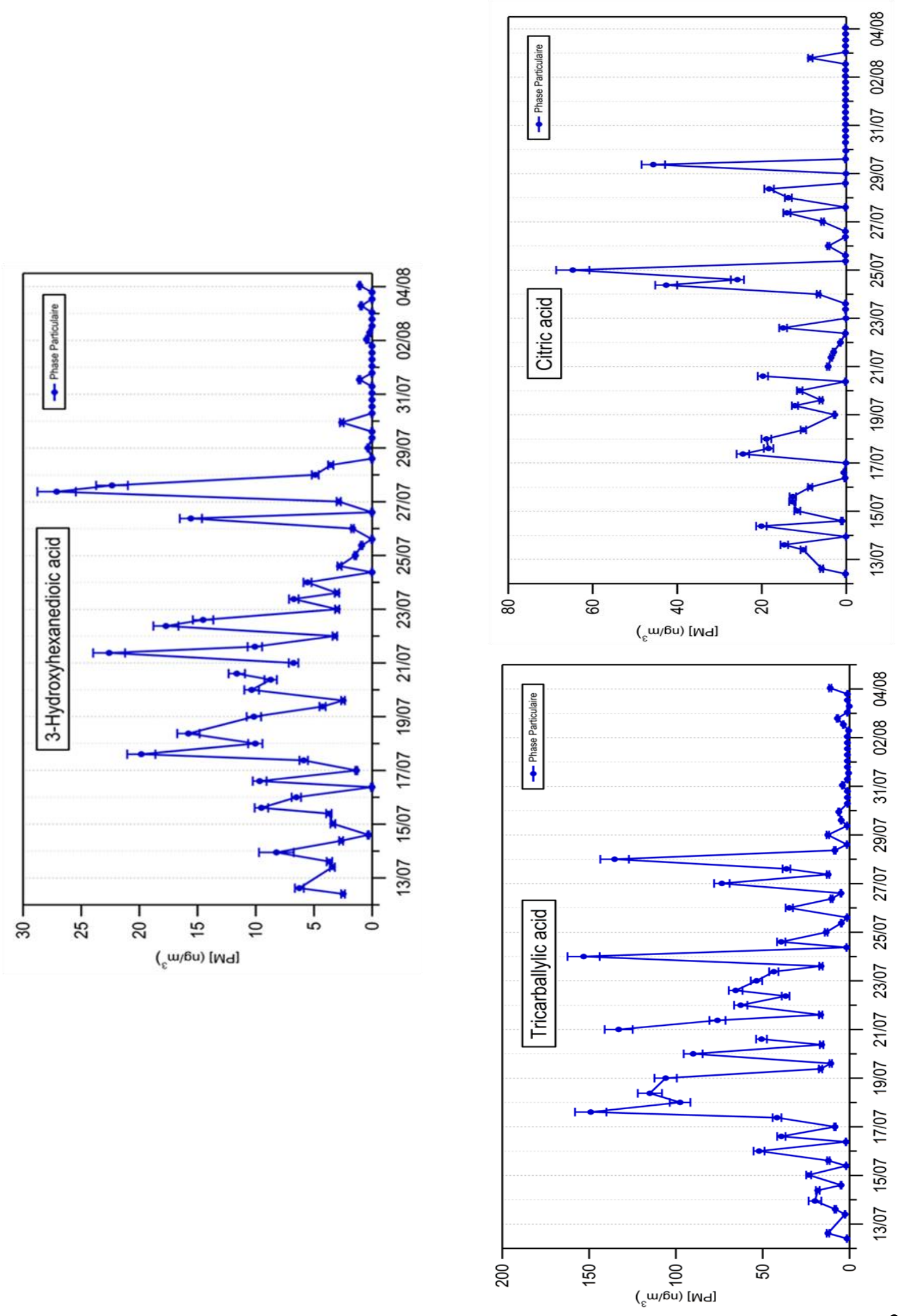


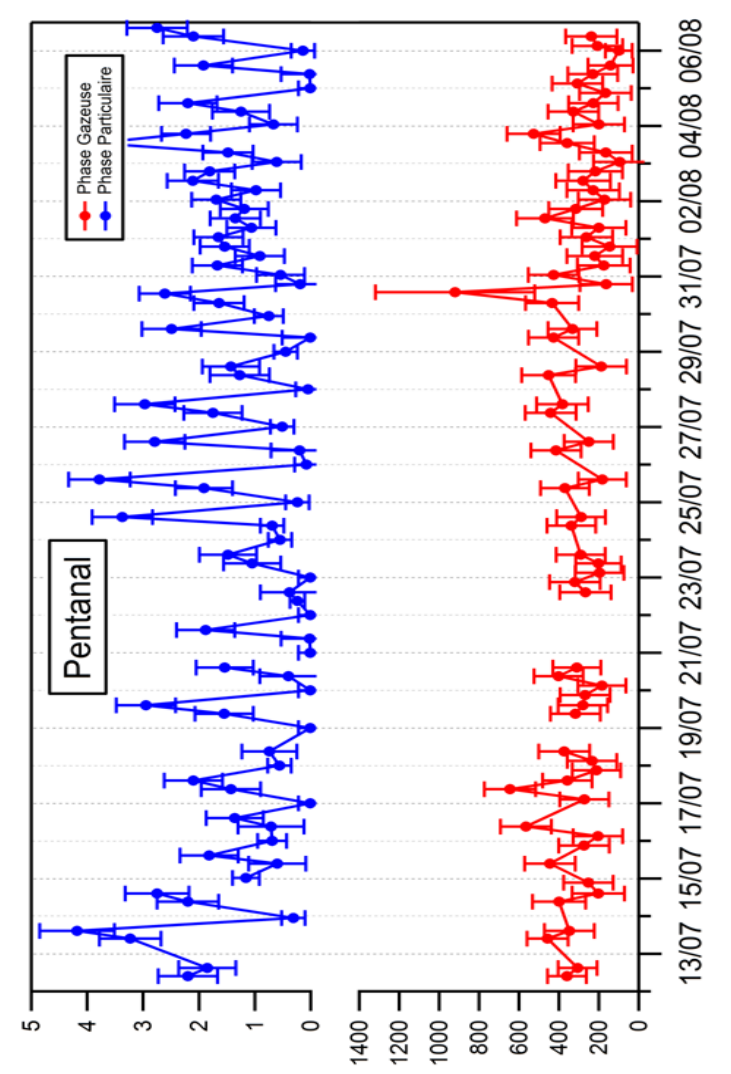

$\left({ }_{\varepsilon} w / 6 u\right)[W d]$

$(\varepsilon / 6 u)$ [zeg]

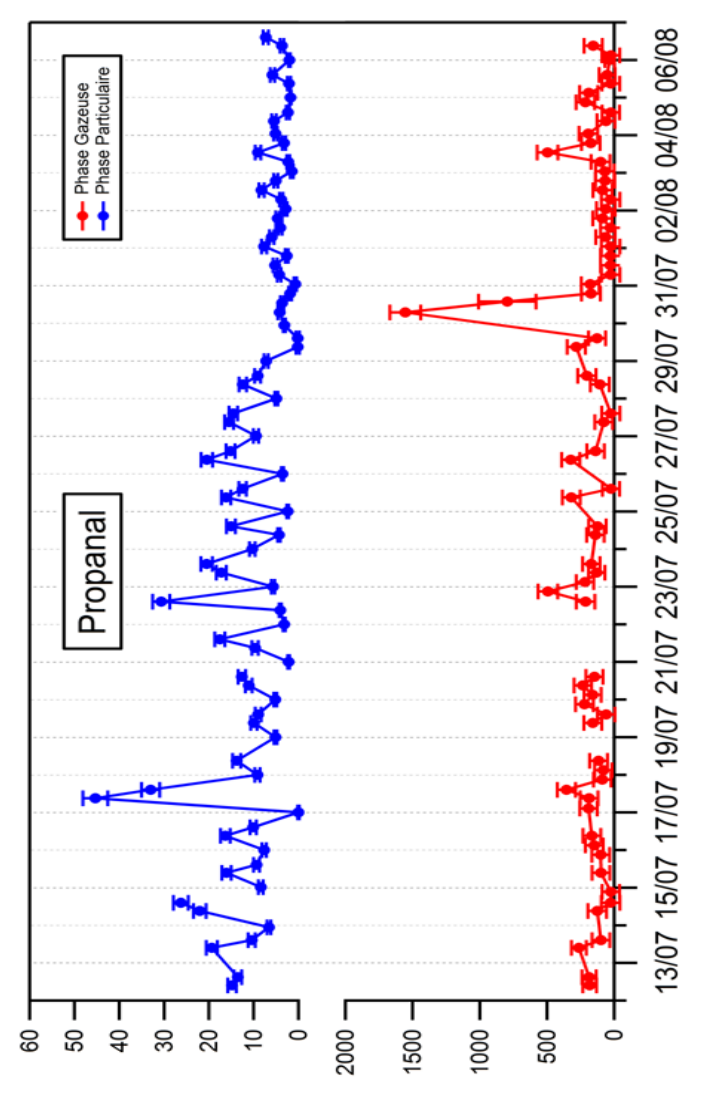

$\left({ }_{\varepsilon} w / 6 u\right)[W d]$

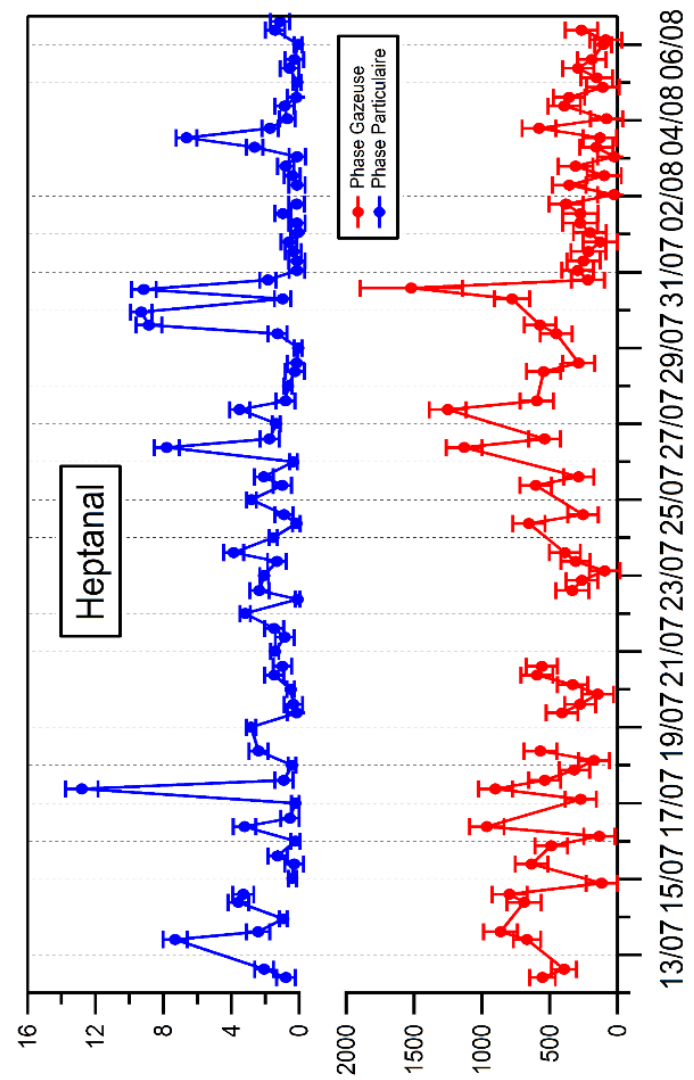

$\left({ }_{\varepsilon} m / 6 u\right)[W d] \quad\left({ }_{\varepsilon} w / 6 u\right)[z e \fallingdotseq]$

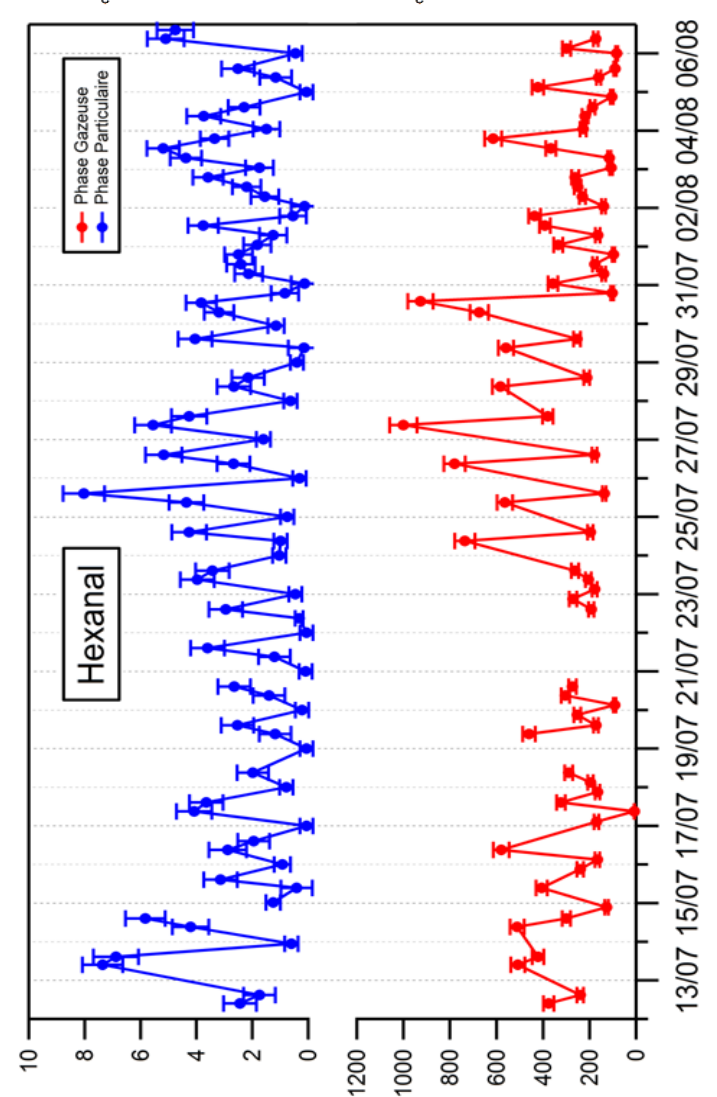

$\left({ }_{c} w / 6 u\right)[W d] \quad(c w / 6 u)[z e g]$ 

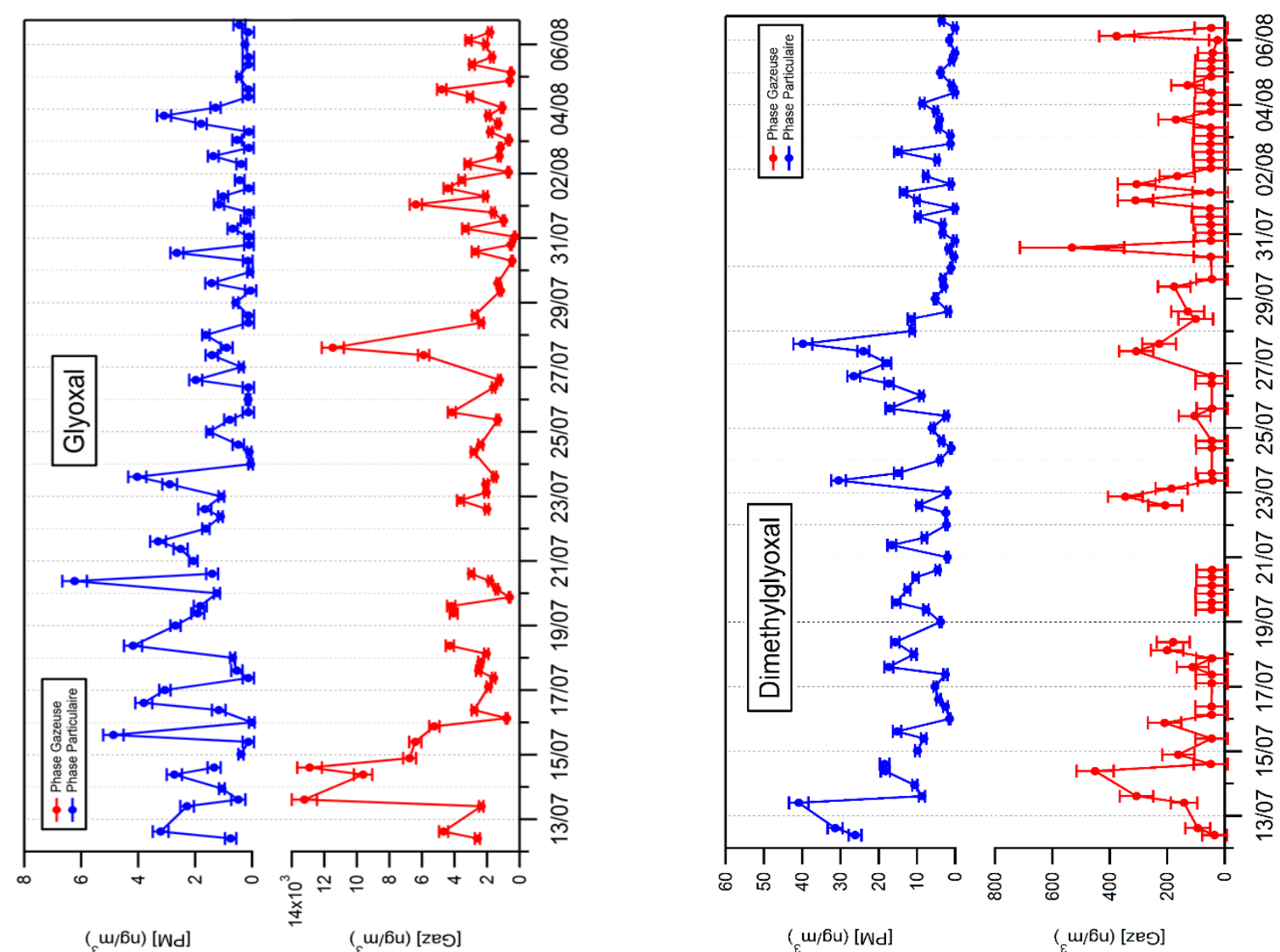

(w/bu) [Wd]

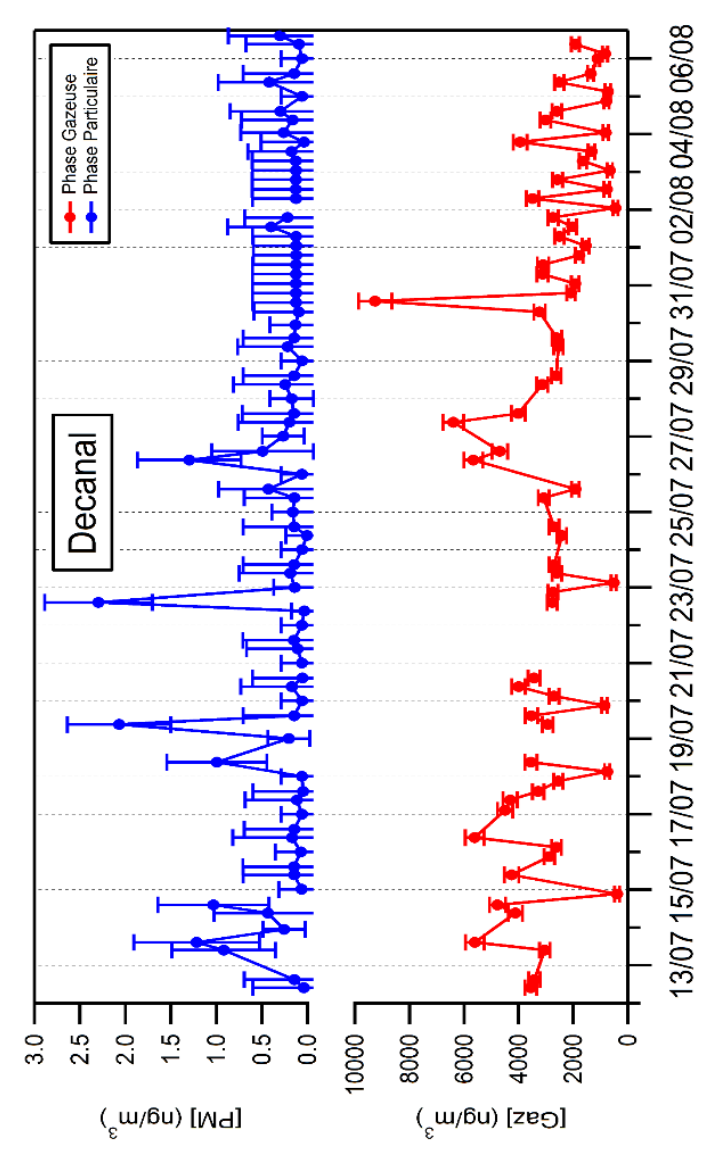

$\left({ }_{\varepsilon} m / 6 u\right)[N d] \quad\left(w_{\varepsilon} w / 6 u\right)[z e g]$

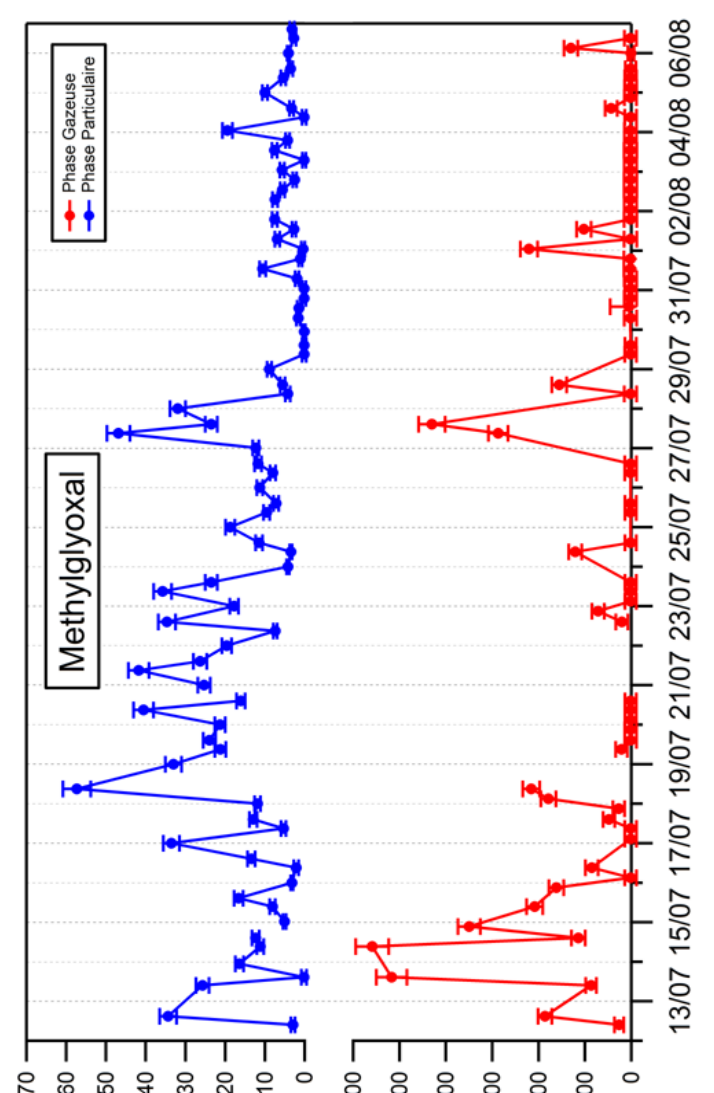

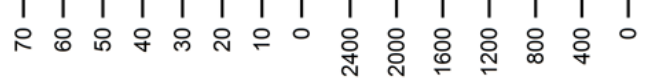

$\left({ }_{\varepsilon} w / 6 u\right)[W d] \quad\left({ }_{\varepsilon} w / 6 u\right)[z e g]$ 


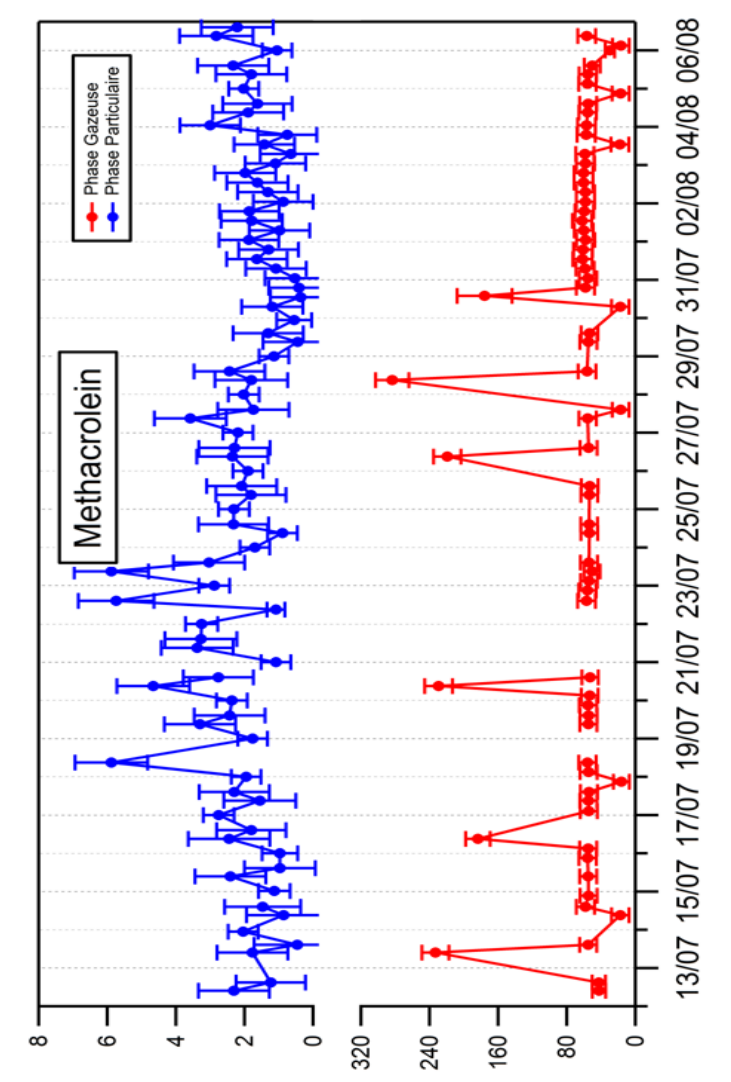

( $w / 6 \mathrm{bu}$ [zeg]

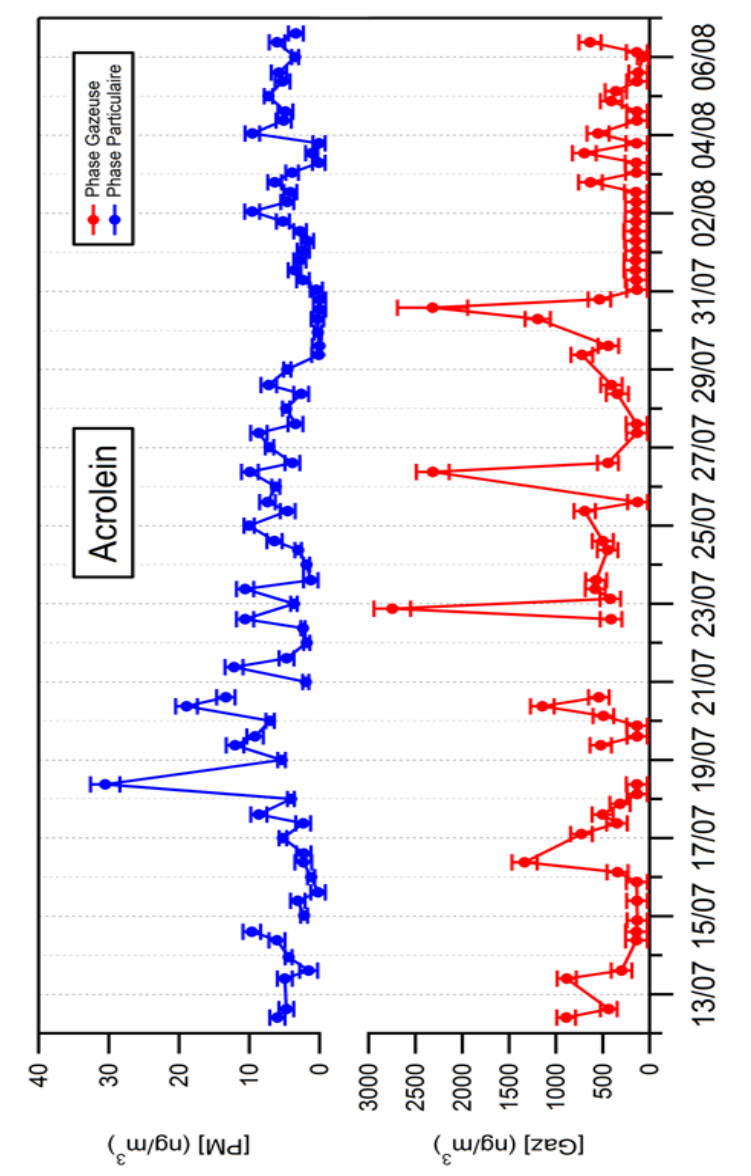

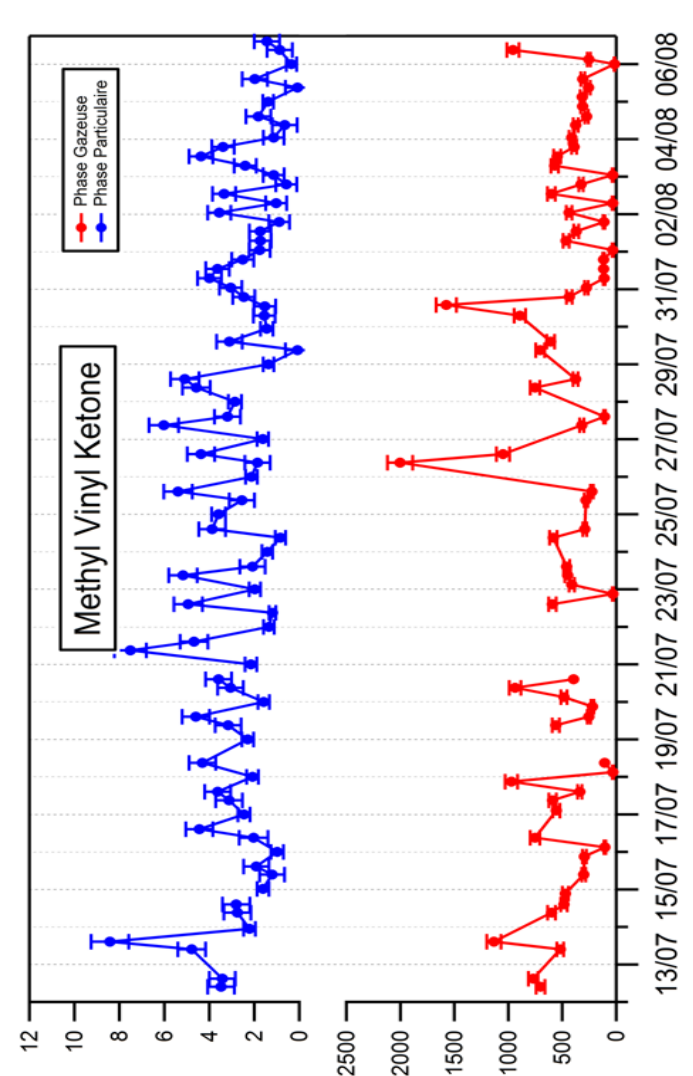

$\left({ }_{\varepsilon} w / 6 u\right)[W d]$

( $u / 6 u$ ) [zeg]

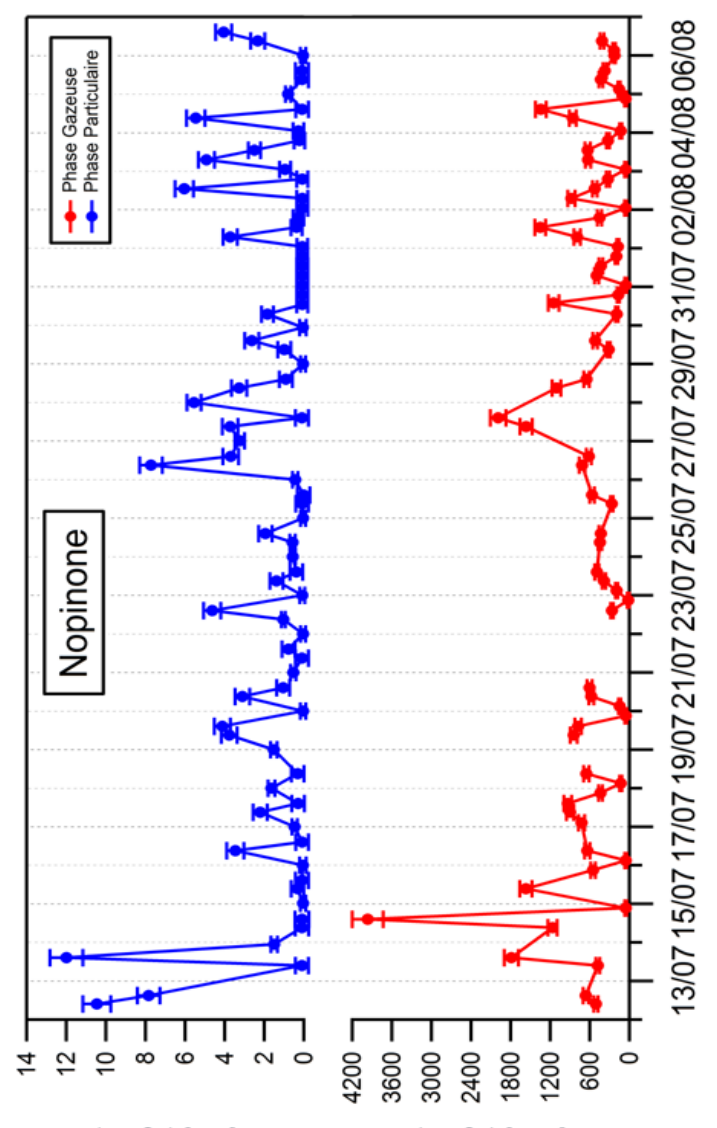

$\left({ }_{\varepsilon} w / 6 u\right)[W d]$

( $\left.{ }_{\varepsilon} w / 6 u\right)$ [zeo] 

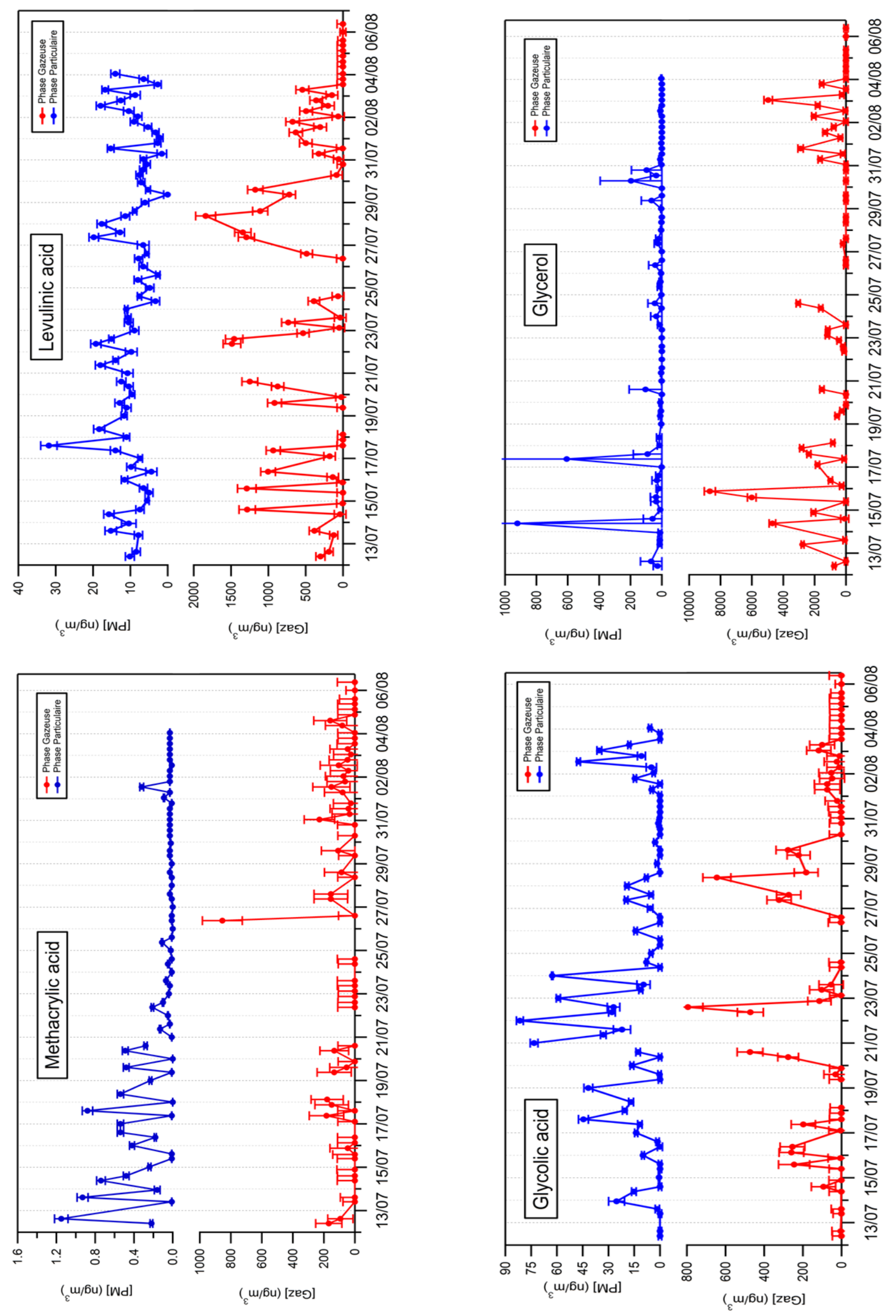
Supplementary material 3: Time series of MSA (methanesulfonic acid, black line) and water soluble HULIS (red line) during ChArMEx campaign.

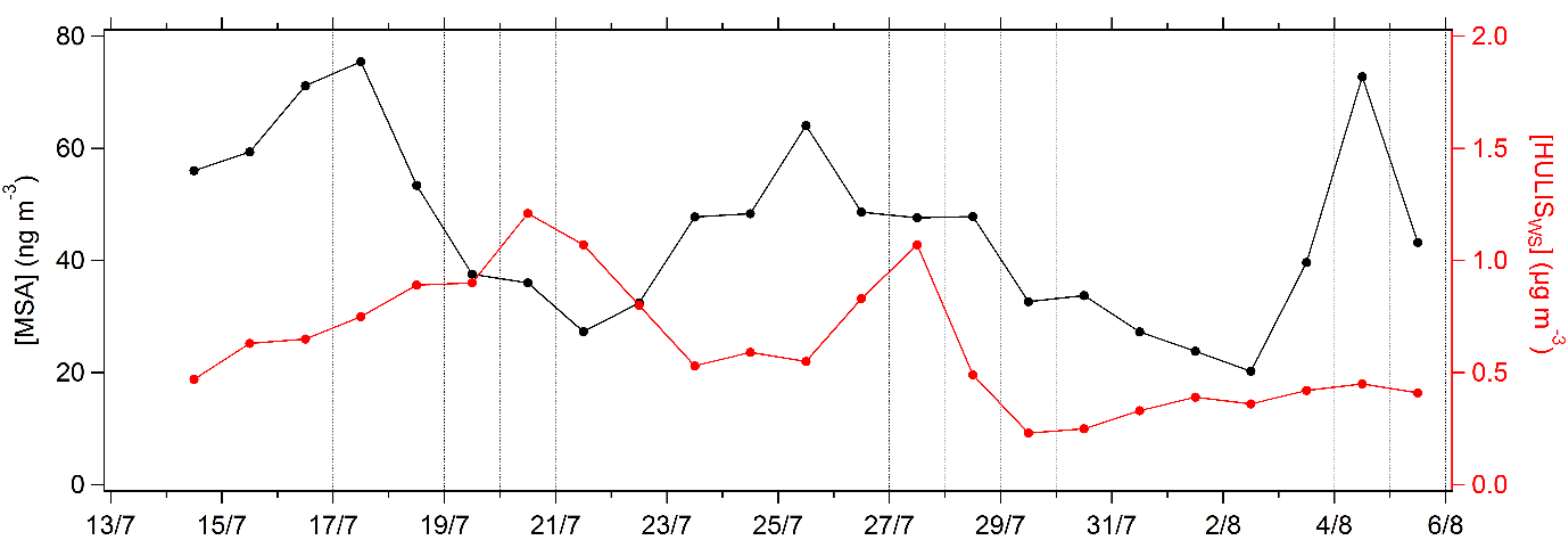

